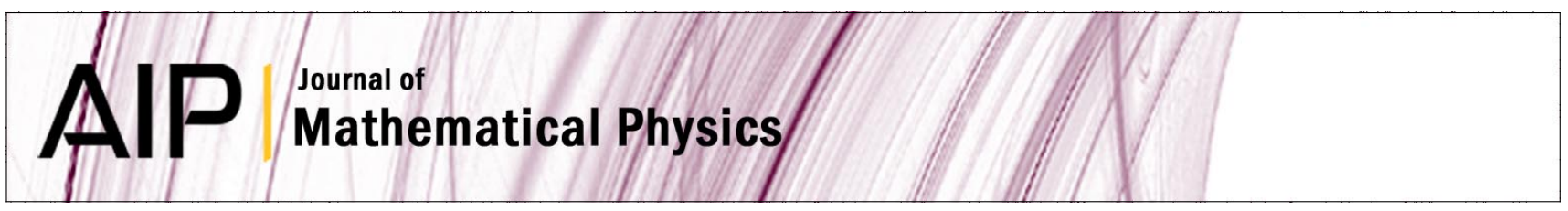

\title{
Quasifinite representations of classical Lie subalgebras of $W_{\infty}, p$
}

José I. García and José I. Liberati

Citation: J. Math. Phys. 54, 073502 (2013); doi: 10.1063/1.4812556

View online: http://dx.doi.org/10.1063/1.4812556

View Table of Contents: http://jmp.aip.org/resource/1/JMAPAQ/v54/i7

Published by the AIP Publishing LLC.

Additional information on J. Math. Phys.

Journal Homepage: http://jmp.aip.org/

Journal Information: http://jmp.aip.org/about/about_the_journal

Top downloads: http://jmp.aip.org/features/most_downloaded

Information for Authors: http://jmp.aip.org/authors

\section{ADVERTISEMENT}

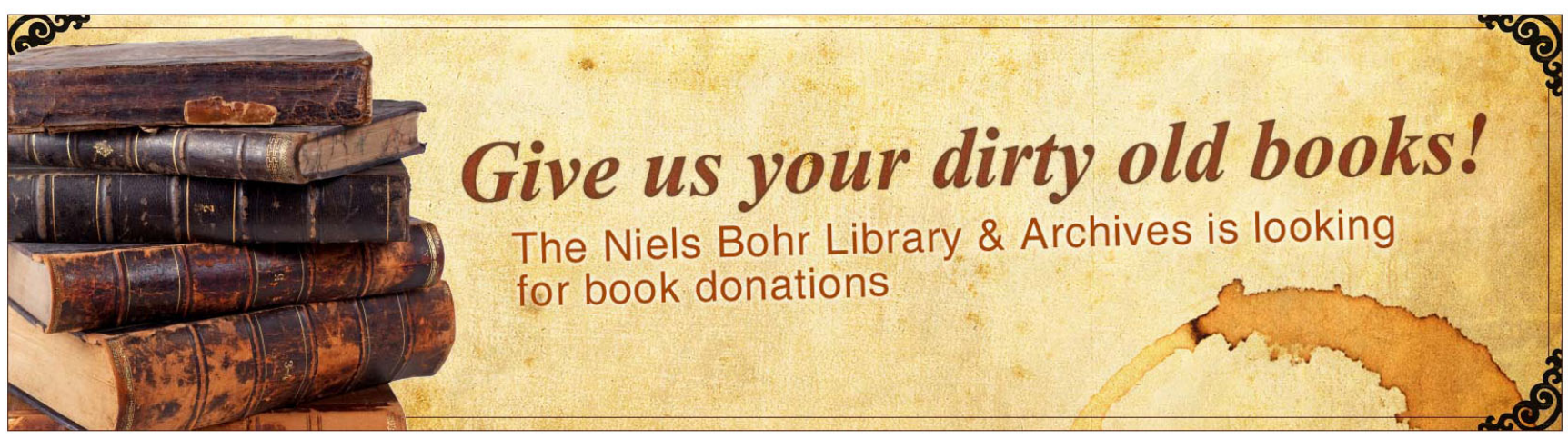




\title{
Quasifinite representations of classical Lie subalgebras of $W_{\infty, p}$ \\ José I. García ${ }^{\mathrm{a})}$ and José I. Liberatib) \\ Famaf-Ciem, Univ. Nac. Córdoba, Ciudad Universitaria, 5000 Córdoba, Argentina
}

(Received 10 August 2012; accepted 13 June 2013; published online 2 July 2013)

\begin{abstract}
We show that there are exactly two anti-involutions $\sigma_{ \pm}$of the algebra of differential operators on the circle that are a multiple of $p\left(t \partial_{t}\right)$ preserving the principal gradation $(p \in \mathbb{C}[x]$ non-constant). We classify the irreducible quasifinite highest weight representations of the central extension $\widehat{\mathcal{D}}_{p}^{ \pm}$of the Lie subalgebra fixed by $-\sigma_{ \pm}$. The most important cases are the subalgebras $\widehat{\mathcal{D}}_{x}^{ \pm}$of $W_{\infty}$ that are obtained when $p(x)$ $=x$. In these cases, we realize the irreducible quasifinite highest weight modules in terms of highest weight representation of the central extension of the Lie algebra of infinite matrices with finitely many nonzero diagonals over the algebra $\mathbb{C}[u] /\left(u^{m+1}\right)$ and its classical Lie subalgebras of $C$ and D types. () 2013 AIP Publishing LLC. [http://dx.doi.org/10.1063/1.4812556]
\end{abstract}

\section{INTRODUCTION}

The universal central extension $\widehat{\mathcal{D}}$ of the Lie algebra of differential operators on the circle (described first in Ref. 7) is usually denoted by physicists as $W_{1+\infty}$, and it is one of the $\mathcal{W}$-infinity algebras that naturally arise in various physical theories, such as conformal field theory, the theory of quantum Hall effect, etc.

The difficulty in understanding the representation theory of a Lie algebra of this kind is that although it admits a $\mathbb{Z}$-gradation (and thus the associated triangular decomposition), each of the graded subspaces is still infinite dimensional, and therefore the study of highest weight modules with the finiteness requirement on the dimensions of their graded subspaces (which we will refer to as quasifiniteness condition) becomes a non-trivial problem.

The study of quasifinite highest weight modules of $\widehat{\mathcal{D}}$ was initiated by Kac and Radul. ${ }^{8}$ They were able to give a characterization of its irreducible quasifinite highest weight representations and these modules were constructed in terms of modules of the Lie algebra $\widehat{\mathfrak{g l}}_{\infty}^{[m]}$ which is the central extension of the Lie algebra $\mathfrak{g}_{\infty}^{[m]}$ of infinite matrices with finitely many nonzero diagonals taking values in the truncated polynomial algebra $R_{m}=\mathbb{C}[u] /\left(u^{m+1}\right)$. On the basis of this analysis, further studies were made within the framework of vertex algebra theory for the $\widehat{\mathcal{D}}$ algebra, ${ }^{5,9}$ and for its matrix version. ${ }^{3}$ In Ref. 6 a general approach to the theory of quasifinite highest weight modules over $\mathbb{Z}$-graded Lie algebras was developed, which makes the basic ideas of Ref. 8 much clearer, and these general results will be applied here. In Refs. 1 and 6, they develop the theory of quasifinite highest weight representations of the subalgebras $\widehat{\mathcal{D}}_{p}$ of $\widehat{\mathcal{D}}$, also known in the literature as $W_{\infty, p}$, where $\widehat{\mathcal{D}}_{p}(p \in \mathbb{C}[x])$ is the central extension of the Lie algebra $\mathcal{D} p\left(t \partial_{t}\right)$ of differential operators on the circle that are a multiple of $p\left(t \partial_{t}\right)$. The most important of these subalgebras is $W_{\infty}=\widehat{\mathcal{D}}_{x}$ that is obtained by taking $p(x)=x$. Classical Lie subalgebras of $\widehat{\mathcal{D}}$ appear by the study of anti-involutions on $\widehat{\mathcal{D}}$. The orthogonal subalgebras of $\widehat{\mathcal{D}}$ were studied in Ref. 10. The symplectic subalgebra of $\widehat{\mathcal{D}}$ was considered in Ref. 2 in relation to number theory, and the representation theory was developed in Ref. 4.

\footnotetext{
a) joseigarciamath@gmail.com

b) liberati@mate.uncor.edu and joseliberati@gmail.com
} 
The idea of the present work is to extend some results from Ref. 10 to the family of subalgebras $\widehat{\mathcal{D}}_{p}$. More precisely, in Sec. II we show that there are exactly two, up to conjugation, anti-involutions $\sigma_{ \pm}$of $\mathcal{D}_{p}^{a}$ preserving the principal gradation. In Sec. III, we classify the irreducible quasifinite highest weight representations of the central extension $\widehat{\mathcal{D}}_{p}^{ \pm}$of the Lie subalgebra of $\widehat{\mathcal{D}}_{p}$ fixed by $-\sigma_{ \pm}$. In particular, if $p=1$, from our results we recover several theorems obtained in Ref. 10 . The other most important cases are the subalgebras $\widehat{\mathcal{D}}_{x}^{ \pm}$of $W_{\infty}$ that are obtained by taking $p(x)=x$. For these cases, in Sec. IV we study the interplay between $\widehat{\mathcal{D}}_{x}^{ \pm}$and some subalgebras of $\widehat{\mathfrak{g l}}_{\infty}^{[m]}$, and in Sec. V we realize the irreducible quasifinite highest weight representations in terms of the highest weight modules of the Lie algebra $\widehat{\mathfrak{g l}}_{\infty}^{[\mathrm{m}]}$ and its classical Lie subalgebras of $C$ and $D$ types. Observe that the symplectic subalgebra of $\mathcal{D}$ considered in Refs. 2 and 4 is a particular case of our general results, in that it corresponds to $\widehat{\mathcal{D}}_{x}^{+}$.

\section{ANTI-INVOLUTION OF $\mathcal{D}_{p}^{a}$ PRESERVING ITS PRINCIPAL GRADATION}

Let $\mathcal{D}^{a}$ be the associative algebra of regular differential operators on the circle, i.e., the operators on $\mathbb{C}\left[t, t^{-1}\right]$ of the form

$$
E=e_{k}(t) \partial_{t}^{k}+e_{k-1}(t) \partial_{t}^{k-1}+\cdots+e_{0}(t), \text { where } e_{i}(t) \in \mathbb{C}\left[t, t^{-1}\right],
$$

the elements

$$
J_{k}^{l}=-t^{k+l} \partial_{t}^{l} \quad\left(l \in \mathbb{Z}_{+}, k \in \mathbb{Z}\right)
$$

form its basis, where $\partial_{t}$ denotes $\frac{d}{d t}$. Another basis of $\mathcal{D}^{a}$ is

$$
L_{k}^{l}=-t^{k} D^{l} \quad\left(l \in \mathbb{Z}_{+}, k \in \mathbb{Z}\right),
$$

where $D=t \partial_{t}$. Let $\mathcal{D}$ denote the Lie algebra obtained from $\mathcal{D}^{a}$ by taking the usual bracket, i.e.,

$$
\left[t^{r} f(D), t^{s} g(D)\right]=t^{r+s}(f(D+s) g(D)-f(D) g(D+r)),
$$

where $f, g \in \mathbb{C}[x]$ and $s, t \in \mathbb{Z}$. Given $p \in \mathbb{C}[x]$, consider the following family of subalgebras of $\mathcal{D}^{a}$ :

$$
\mathcal{D}_{p}^{a}:=\mathcal{D}^{a} p(D),
$$

and denote by $\mathcal{D}_{p}$ the associated Lie algebra (cf. Ref. 6).

Letting $w t t^{k} f(D)=k$ defines the principal $\mathbb{Z}$-gradation of $\mathcal{D}^{a}$ and $\mathcal{D}_{p}^{a}$ :

$$
\mathcal{D}_{p}^{a}=\bigoplus_{j \in \mathbb{Z}}\left(\mathcal{D}_{p}^{a}\right)_{j}, \text { where }\left(\mathcal{D}_{p}^{a}\right)_{j}=\left\{t^{j} f(D) p(D): f \in \mathbb{C}[x]\right\} .
$$

An anti-involution $\sigma$ of $\mathcal{D}_{p}^{a}$ is an involutive anti-automorphism of $\mathcal{D}_{p}^{a}$, i.e., $\sigma: \mathcal{D}_{p}^{a} \rightarrow \mathcal{D}_{p}^{a}$ with $\sigma^{2}=I d, \sigma(b X+Y)=b \sigma(X)+\sigma(Y)$, and $\sigma(X Y)=\sigma(Y) \sigma(X)$, where $X, Y \in \mathcal{D}_{p}^{a}, b \in \mathbb{C}$.

The main result of this section is the following theorem with the classification of all antiinvolutions of $\mathcal{D}_{p}^{a}$ that preserve the principal $\mathbb{Z}$-gradation.

Theorem 2.1. Let $p \in \mathbb{C}[x]$ be a nonzero polynomial. There exists an anti-involution in $\mathcal{D}_{p}^{a}$ that preserves the principal $\mathbb{Z}$-gradation if and only if there exists $c \in \mathbb{C}$ such that $p(x)=\varepsilon p(-x+c)$, where $\varepsilon=(-1)^{\operatorname{deg}(p)}$.

If $\operatorname{deg}(p) \geq 1$, then $c$ is unique and there exist only two anti-involutions given by

$$
\sigma_{ \pm}\left(t^{k} f(D) p(D)\right)=\varepsilon( \pm t)^{k} f(-D-k+c) p(D) .
$$

If $\operatorname{deg}(p)=0$, then $c$ is a free parameter, and there are only two families of anti-involutions given by (2.1).

Remark 2.2. When deg $(p)=0$, we recover the classification obtained in Proposition $2.1 .{ }^{10}$

In the last part of this section, we present the proof of Theorem 2.1 throughout several lemmas. 
Let $\sigma: \mathcal{D}_{p}^{a} \rightarrow \mathcal{D}_{p}^{a}$ be an anti-involution that preserves the principal gradation. Then $\sigma$ induces a map $\sigma_{0}: \mathcal{D}^{a} \rightarrow \mathcal{D}^{a}$ as follows:

$$
\sigma\left(t^{k} f(D) p(D)\right)=\sigma_{0}\left(t^{k} f(D)\right) p(D) .
$$

It is clear that $\sigma_{0}$ preserves the principal gradation and furthermore, the characterization of $\sigma$ is equivalent to the characterization of $\sigma_{0}$.

Lemma 2.3. Let $f, g \in \mathbb{C}[D]$ and $k, m \in \mathbb{Z}$. Then

(a) $\sigma_{0}$ is $\mathbb{C}$-linear;

(b) $\sigma_{0}^{2}=I d$

(c) $\quad \sigma_{0}\left(t^{k+m} f(D+m) p(D+m) g(D)\right)=\sigma_{0}\left(t^{m} g(D)\right) p(D) \sigma_{0}\left(t^{k} f(D)\right)$;

(d) $\quad \sigma_{0}(f(D) g(D) p(D))=\sigma_{0}(f(D)) \sigma_{0}(g(D)) p(D)$.

Proof. Using that $\sigma$ is an anti-involution, (a) and (b) follow immediately. For $f, g \in$ $\mathbb{C}[D], k, m \in \mathbb{Z}$, we have

$$
\sigma\left(t^{k} f(D) p(D) t^{m} g(D) p(D)\right)=\sigma_{0}\left(t^{m} g(D)\right) p(D) \sigma_{0}\left(t^{k} f(D)\right) p(D)
$$

and

$$
\sigma\left(t^{k} f(D) p(D) t^{m} g(D) p(D)\right)=\sigma_{0}\left(t^{k+m} f(D+m) p(D+m) g(D)\right) p(D)
$$

obtaining (c). Observe that (d) follows from (c) since $\left(\mathcal{D}^{a}\right)_{0}$ is an Abelian subalgebra of $\mathcal{D}^{a}$ and $\sigma_{0}$ preserves the gradation, finishing the proof.

We shall need the following notation: $\sigma_{0}\left(t^{k}\right)=t^{k} \varepsilon_{k}$, with $\varepsilon_{k}$ in $\mathbb{C}[D]$.

Lemma 2.4. (a) For all $k \in \mathbb{Z}$, we have $\varepsilon_{k}= \pm 1$.

(b) There exists $c \in \mathbb{C}$ such that for all $k \in \mathbb{Z}$ and $f \in \mathbb{C}[D]$, we have

$$
\sigma_{0}\left(t^{k} f(D)\right)=\varepsilon_{k} t^{k} f(-D-k+c) .
$$

Proof. Using Lemma 2.3(d) with $f=g=1$, we have

$$
\sigma_{0}(p(D))=\varepsilon_{0}^{2} p(D)
$$

then by Lemma 2.3(b) and (2.5), $p(D)=\sigma_{0}\left(\varepsilon_{0}^{2} p(D)\right)$ and using again Lemma 2.3(d) with $f=1$ and $g=\varepsilon_{0}^{2}$, we obtain

$$
p(D)=\varepsilon_{0} \sigma_{0}\left(\varepsilon_{0}^{2}\right) p(D) .
$$

Then from (2.6), $1=\varepsilon_{0} \sigma_{0}\left(\varepsilon_{0}^{2}\right)$ therefore $\varepsilon_{0}$ is a constant. Moreover, by Lemmas 2.3(a) and (b), we have $1=\sigma_{0}^{2}(1)=\varepsilon_{0}^{2}$, obtaining

$$
\varepsilon_{0}= \pm 1
$$

Now we shall prove that

$$
\sigma_{0}\left(t^{l} D^{i}\right)=t^{l} \varepsilon_{l}\left(\varepsilon_{0} \sigma_{0}(D)-l\right)^{i} \text { for all } l \in \mathbb{Z} \text {, and } i \in \mathbb{Z}_{+},
$$

by using induction in $i$. The case $i=0$ follows by notation. Now, using Lemma 2.3(c) with $t^{k} f(D)=$ $(D-l), t^{m} g(D)=t^{l} D^{i}$, we have

$$
\begin{aligned}
\sigma_{0}\left(t^{l} D^{i+1} p(D+l)\right) & =\sigma_{0}\left(t^{l} D^{i}\right) p(D) \sigma_{0}(D-l) \\
& =t^{l} \varepsilon_{l}\left(\varepsilon_{0} \sigma_{0}(D)-l\right)^{i} p(D)\left(\sigma_{0}(D)-\varepsilon_{0} l\right) ;
\end{aligned}
$$


on the other hand, using again Lemma 2.3(c) with $t^{k} f(D)=1, t^{m} g(D)=t^{l} D^{i+1}$, we obtain

$$
\sigma_{0}\left(t^{l} D^{i+1} p(D+l)\right)=\sigma_{0}\left(t^{l} D^{i+1}\right) p(D) \varepsilon_{0} .
$$

Comparing (2.9) with (2.10), and using (2.7), we obtain (2.8).

Note that, given $f \in \mathbb{C}[x]$ and using the linearity of $\sigma_{0}$ together with (2.8), we have

$$
\sigma_{0}\left(t^{k} f(D)\right)=t^{k} \varepsilon_{k} f\left(\varepsilon_{0} \sigma_{0}(D)-k\right) .
$$

Since $\sigma_{0}$ preserves the $\mathbb{Z}$-gradation, we can assume that $\sigma_{0}(D)=g(D)$ for some $g \in \mathbb{C}[x]$. Then by Lemma 2.3(b) and (2.11), we have $D=\sigma_{0}^{2}(D)=\varepsilon_{0} g\left(\varepsilon_{0} g(D)\right)$. Using (2.7), it follows that $\operatorname{deg}(g)^{2}=1$ and therefore $\operatorname{deg}(g)=1$. Then $\sigma_{0}(D)=a D+b$ for some $a, b \in \mathbb{C}$ with $a \neq 0$. Finally,

$$
D=\sigma_{0}^{2}(D)=\sigma_{0}(a D+b)=a^{2} D+\left(a+\varepsilon_{0}\right) b,
$$

obtaining

$$
\sigma_{0}(D)=a D+b \text { with } a= \pm 1,\left(a+\varepsilon_{0}\right) b=0 .
$$

Using Lemma 2.3(b) and (2.11), for all $k \in \mathbb{Z}$,

$$
t^{k}=\sigma_{0}^{2}\left(t^{k}\right)=\sigma_{0}\left(t^{k} \varepsilon_{k}\right)=t^{k} \varepsilon_{k}(D) \varepsilon_{k}\left(\varepsilon_{0} \sigma_{0}(D)-k\right),
$$

then $\operatorname{deg}\left(\varepsilon_{k}\right)=0$ and furthermore $\varepsilon_{k}^{2}=1$, finishing the proof of (a).

Observe that, using (2.11), (2.12), and Lemma 2.4(a), we have

$$
\sigma_{0}\left(t^{k} f(D)\right)=\varepsilon_{k} t^{k} f\left(\varepsilon_{0} a D-k+c\right), \text { where } c=\varepsilon_{0} b .
$$

Hence, in order to finish the proof of (b), it remains to see that $\varepsilon_{0} a=-1$. But

$$
\begin{aligned}
t^{k} D & =\sigma_{0}^{2}\left(t^{k} D\right) \\
& =\sigma_{0}\left(\varepsilon_{k} t^{k}\left(\varepsilon_{0} a D-k+\varepsilon_{0} b\right)\right) \\
& =t^{k} D-\left(\varepsilon_{0} a+1\right) k t^{k}
\end{aligned}
$$

for all $k$ in $\mathbb{Z}$, therefore $\varepsilon_{0} a+1=0$.

Proof of Theorem 2.1. Let $\sigma: \mathcal{D}_{p}^{a} \rightarrow \mathcal{D}_{p}^{a}$ be an anti-involution that preserves the principal $\mathbb{Z}$-gradation. From (2.2) and Lemma 2.4(b)

$$
\sigma\left(t^{k} f(D) p(D)\right)=\varepsilon_{k} t^{k} f(-D-k+c) p(D)
$$

for some $c \in \mathbb{C}$.

Moreover, from (2.5) and Lemma 2.4(a) we obtain $\sigma_{0}(p(D))=p(D)$. Then by Lemma 2.4(b), we have that $p$ must satisfy

$$
p(D)=\varepsilon_{0} p(-D+c) \text { for some } c \in \mathbb{C} .
$$

If $n=\operatorname{deg}(p)>0$, by considering the coefficients of $D^{n}$ and $D^{n-1}$ in both sides of (2.15), we have $\varepsilon_{0}=(-1)^{n}$ and $c=-\frac{2 c_{n-1}}{n c_{n}}$, respectively, where $p(x)=\sum_{i=0}^{n} c_{i} x^{i}$, so $c$ is totally determined by the coefficients of $p$.

If $\operatorname{deg}(p)=0$, using (2.15) we get $\varepsilon_{0}=1$ and $c$ is a free parameter.

On the other hand,

$$
\sigma\left(t^{k} p(D) t^{m} p(D)\right)=\sigma\left(t^{m} p(D)\right) \sigma\left(t^{k} p(D)\right)
$$

with

$$
\begin{aligned}
\sigma\left(t^{k} p(D) t^{m} p(D)\right) & =\sigma\left(t^{k+m} p(D+m) p(D)\right) \\
& =\varepsilon_{k+m} t^{k+m} p(-D-k+c) p(D) \\
& =\varepsilon_{0} \varepsilon_{k+m} t^{k+m} p(D+k) p(D)
\end{aligned}
$$


and

$$
\begin{aligned}
\sigma\left(t^{m} p(D)\right) \sigma\left(t^{k} p(D)\right) & =\varepsilon_{m} \varepsilon_{k} t^{m} p(D) t^{k} p(D) \\
& =\varepsilon_{m} \varepsilon_{k} t^{k+m} p(D+k) p(D) .
\end{aligned}
$$

From (2.17) and (2.18) we have that (2.16) holds if and only if

$$
\varepsilon_{k+m}=\varepsilon_{0} \varepsilon_{k} \varepsilon_{m},
$$

and this is true for all $k, m \in \mathbb{Z}$. Then, if we take $k=1$ and $m=-1$ from (2.19) and Lemma 2.4(a), we have that $\varepsilon_{1}=\varepsilon_{-1}$ and by induction

$$
\varepsilon_{k}=\varepsilon_{0}\left(\varepsilon_{0} \varepsilon_{1}\right)^{k} \text { for all } k \in \mathbb{Z} .
$$

Since $\varepsilon_{0}$ is totally determined, we could have only two anti-involutions depending on the choice of $\varepsilon_{1}$. Using (2.14) and (2.20), we have the following cases:

- if $\varepsilon_{1}=\varepsilon_{0}$, then $\sigma\left(t^{k} f(D) p(D)\right)=\varepsilon_{0} t^{k} f(-D-k+c) p(D)$;

- if $\varepsilon_{1}=-\varepsilon_{0}$, then $\sigma\left(t^{k} f(D) p(D)\right)=\varepsilon_{0}(-t)^{k} f(-D-k+c) p(D)$.

Conversely, it is straightforward to check that if $p$ satisfies (2.15) then the two previous cases are anti-involutions, finishing the proof.

\section{QUASIFINITE HIGHEST-WEIGHT MODULES OVER $\widehat{\mathcal{D}}_{p}^{ \pm}$}

Let $p \in \mathbb{C}[x]$ with $n=\operatorname{deg}(p)$ that satisfies Theorem 2.1, i.e., $p(x)=(-1)^{n} p(-x+c)$ for some $c \in \mathbb{C}$. We denote by $\mathcal{D}_{p}^{ \pm}$the Lie subalgebra of $\mathcal{D}_{p}$ consisting of its minus $\sigma_{ \pm}$-fixed points, i.e.,

$$
\mathcal{D}_{p}^{ \pm}=\left\{d \in \mathcal{D}_{p}: \sigma_{ \pm}(d)=-d\right\}
$$

It inherits a $\mathbb{Z}$-gradation from $\mathcal{D}_{p}$ since $\sigma_{ \pm}$preserves the principal $\mathbb{Z}$-gradation of $\mathcal{D}_{p}$, then $\mathcal{D}_{p}^{ \pm}=$ $\bigoplus_{k \in \mathbb{Z}}\left(\mathcal{D}_{p}^{ \pm}\right)_{k}$, where

$$
\left(\mathcal{D}_{p}^{ \pm}\right)_{k}=\left\{t^{k} f(D) p(D): f \in \mathbb{C}[x] \text { and } \sigma_{ \pm}\left(t^{k} f(D) p(D)\right)=-t^{k} f(D) p(D)\right\} .
$$

Let us denote by $\mathbb{C}[x]^{(0)}$ (respectively $\mathbb{C}[x]^{(1)}$ ) the set of all even (respectively odd) polynomials in $\mathbb{C}[x]$. Also, we let $\bar{k}=0$ if $k$ is an odd integer and $\bar{k}=1$ if $k$ is even. The following lemma gives a complete description of $\left(\mathcal{D}_{p}^{ \pm}\right)_{k}$.

Lemma 3.1. (a) $\left(\mathcal{D}_{p}^{+}\right)_{k}=\left\{t^{k} f\left(D-\frac{c-k}{2}\right) p(D): f \in \mathbb{C}[x]^{(\bar{n})}\right\}$;

(b) $\left(\mathcal{D}_{p}^{-}\right)_{k}=\left\{t^{k} f\left(D-\frac{c-k}{2}\right) p(D): f \in \mathbb{C}[x]^{(\overline{n+k})}\right\}$.

Proof. Let $t^{k} f(D) p(D) \in\left(\mathcal{D}_{p}^{-}\right)_{k}$, then by Theorem 2.1

$$
(-1)^{n+k} t^{k} f(-D-k+c) p(D)=\sigma_{-}\left(t^{k} f(D) p(D)\right)=-t^{k} f(D) p(D)
$$

if and only if $(-1)^{n+k+1} f(x)=f(-x-k+c)$. We define $g(w)=f\left(w+\frac{c-k}{2}\right)$ and for $x=$ $w-\frac{c-k}{2}$, we have $g(-x)=f(-w-k+c)=(-1)^{n+k+1} f(w)=(-1)^{n+k+1} f\left(x+\frac{c-k}{2}\right)=$ $(-1)^{n+k+1} g(x)$, therefore $g(w) \in \mathbb{C}[w]^{(\overline{n+k})}$ and $g\left(x-\frac{c-k}{2}\right)=f(x)$ finishing (b). The proof of (a) is similar. 
Remark 3.2 (Ref. 10).

We have the following two-cocycle on $\mathcal{D}$, where $f(x), g(x) \in \mathbb{C}[x]$

$$
\Psi\left(t^{r} f(D), t^{s} g(D)\right)=\left\{\begin{array}{ll}
\sum_{-r \leqslant m \leqslant-1} f(m) g(m+r) & \text { if } r=-s>0 \\
0 & \text { if } r+s \neq 0 \text { or } r=s=0
\end{array},\right.
$$

and, using skew-symmetry, it is also defined when $r=-s<0$. Denote by $\widehat{\mathcal{D}}$ the central extension of $\mathcal{D}$ by a one-dimensional center $\mathbb{C} C$, corresponding to the two-cocycle $\Psi$, i.e., $\widehat{\mathcal{D}}=\mathcal{D}+\mathbb{C} C$ with the following commutation relation:

$$
\left[t^{r} f(D), t^{s} g(D)\right]=t^{r+s}(f(D+s) g(D)-f(D) g(D+r))+\Psi\left(t^{r} f(D), t^{s} g(D)\right) C .
$$

Denote by $\widehat{\mathcal{D}}_{p}^{ \pm}$the central extension of $\mathcal{D}_{p}^{ \pm}$by $\mathbb{C} C$ corresponding to the restriction of the two-cocycle $\Psi$.

Letting $w t t^{k} f(D) p(D)=k$, wt $C=0$ defines the principal gradation of $\widehat{\mathcal{D}}_{p}^{ \pm}$

$$
\widehat{\mathcal{D}}_{p}^{ \pm}=\bigoplus_{k \in \mathbb{Z}}\left(\widehat{\mathcal{D}}_{p}^{ \pm}\right)_{k}, \quad \text { where } \quad\left(\widehat{\mathcal{D}}_{p}^{ \pm}\right)_{k}=\left(\mathcal{D}_{p}^{ \pm}\right)_{k}+\delta_{0, k} \mathbb{C} C .
$$

In order to apply the general results on quasifinite representations of $\mathbb{Z}$-graded Lie algebras developed in Ref. 6, we need to study the parabolic subalgebras of $\widehat{\mathcal{D}}_{p}^{ \pm}$. Let us recall some general definition and results from Ref. 6.

Let $\mathfrak{g}=\bigoplus_{j \in \mathbb{Z}} \mathfrak{g}_{j}$ be a $\mathbb{Z}$-graded Lie algebra over $\mathbb{C}$, and take $\mathfrak{g}_{+}=\bigoplus_{j>0} \mathfrak{g}_{j}$. A $\mathbb{Z}$-graded subalgebra $\mathfrak{p}$ of $\mathfrak{g}$ is called parabolic if

$$
\mathfrak{p}=\bigoplus_{j \in \mathbb{Z}} \mathfrak{p}_{j}, \text { where } \mathfrak{p}_{j}=\mathfrak{g}_{j} \text { for } j \geq 0 \text { and } \mathfrak{p}_{j} \neq 0 \text { for some } j<0 .
$$

We assume the following properties of $\mathfrak{g}$ :

(P1) $\mathfrak{g}_{0}$ is commutative,

(P2) if $a \in \mathfrak{g}_{-k}(k>0)$ and $\left[a, \mathfrak{g}_{1}\right]=0$, then $a=0$.

Given $a \in \mathfrak{g}_{-1}$ that is nonzero, we define $\mathfrak{p}^{a}=\bigoplus_{j \in \mathbb{Z}} \mathfrak{p}_{j}^{a}$, where $\mathfrak{p}_{j}^{a}=\mathfrak{g}_{j}$ for all $j \geq 0$ and

$$
\mathfrak{p}_{-1}^{a}=\sum\left[\ldots\left[\left[a, \mathfrak{g}_{0}\right], \mathfrak{g}_{0}\right], \ldots\right], \quad \mathfrak{p}_{-k-1}^{a}=\left[\mathfrak{p}_{-1}^{a}, \mathfrak{p}_{-k}^{a}\right] .
$$

It was proved in Ref. 6 that $\mathfrak{p}^{a}$ is the minimal parabolic subalgebra containing $a$.

Definition 3.3. (a) A parabolic subalgebra $\mathfrak{p}$ is called nondegenerate if $\mathfrak{p}_{-j}$ has finite codimension in $\mathfrak{g}_{-j}$ for all $j \in \mathbb{N}$.

(b) An element $a \in \mathfrak{g}_{-1}$ is called nondegenerate if $\mathfrak{p}^{a}$ is nondegenerate.

We will also require the following condition on $\mathfrak{g}$ :

(P3) If $\mathfrak{p}$ is a nondegenerate parabolic subalgebra of $\mathfrak{g}$, then there exists a nondegenerate element $a \in \mathfrak{p}_{-1}$.

A $\mathfrak{g}$-modulo $V$ is called $\mathbb{Z}$-graded if $V=\bigoplus_{j \in \mathbb{Z}} V_{j}$ and $\mathfrak{g}_{i} V_{j} \subset V_{i+j}$. A $\mathbb{Z}$-graded $\mathfrak{g}$-module is called quasifinite if $\operatorname{dim} V_{j}<\infty$ for all $j$.

Given $\lambda \in \mathfrak{g}_{0}^{*}$, a highest-weight module is a $\mathbb{Z}$-graded $\mathfrak{g}$-module $V(\mathfrak{g}, \lambda)$ generated by a highestweight vector $v_{\lambda} \in V(\mathfrak{g}, \lambda)_{0}$ which satisfies

$$
h v_{\lambda}=\lambda(h) v_{\lambda} \quad \text { for } h \in \mathfrak{g} \text { and } \mathfrak{g}_{+} v_{\lambda}=0 .
$$

A nonzero vector $v \in V(\mathfrak{g}, \lambda)$ is called singular if $\mathfrak{g}_{+} v=0$.

The Verma module over $\mathfrak{g}$ is defined as usual:

$$
M(\mathfrak{g}, \lambda)=\mathcal{U}(\mathfrak{g}) \bigotimes_{\mathcal{U}\left(\mathfrak{g}_{0} \oplus \mathfrak{g}_{+}\right)} \mathbb{C}_{\lambda},
$$

where $\mathbb{C}_{\lambda}$ is the one-dimensional ( $\mathfrak{g}_{0} \bigoplus \mathfrak{g}_{+}$)-module given by $h \mapsto \lambda(h)$ if $h \in \mathfrak{g}_{0}, \mathfrak{g}_{+} \mapsto 0$, and the action of $\mathfrak{g}$ is induced by the left multiplication in $\mathcal{U}(\mathfrak{g})$. Here and further $\mathcal{U}(\mathfrak{g})$ stands for the universal 
enveloping algebra of the Lie algebra $\mathfrak{g}$. Any highest weight module $V(\mathfrak{g}, \lambda)$ is a quotient module of $M(\mathfrak{g}, \lambda)$. The irreducible module $L(\mathfrak{g}, \lambda)$ is the quotient of $M(\mathfrak{g}, \lambda)$ by the maximal proper graded submodule.

Consider a parabolic subalgebra $\mathfrak{p}=\bigoplus_{j \in \mathbb{Z}} \mathfrak{p}_{j}$ of $\mathfrak{g}$ and let $\lambda \in \mathfrak{g}_{0}^{*}$ be such that $\left.\lambda\right|_{\mathfrak{g}_{0} \cap[\mathfrak{p}, \mathfrak{p}]}=0$. Then the $\left(\mathfrak{g}_{0} \bigoplus \mathfrak{g}_{+}\right)$-module $\mathbb{C}_{\lambda}$ extends to a $\mathfrak{p}$-module by letting $\mathfrak{p}_{j}$ act as 0 for $j<0$, and we may construct the highest weight module

$$
M(\mathfrak{p}, \mathfrak{g}, \lambda)=\mathcal{U}(\mathfrak{g}) \bigotimes_{\mathcal{U}(\mathfrak{p})} \mathbb{C}_{\lambda}
$$

called the generalized Verma module. Clearly all these highest weight modules are graded. The following result gives the characterization of all irreducible quasifinite highest weight modules.

Theorem 3.4 (Ref. 6). Let $\mathfrak{g}=\bigoplus_{j \in \mathbb{Z}} \mathfrak{g}_{j}$ be a $\mathbb{Z}$-graded Lie algebra over $\mathbb{C}$ that satisfies conditions (P1), (P2), and (P3). The following conditions on $\lambda \in \mathfrak{g}_{0}^{*}$ are equivalent:

(a) $M(\mathfrak{g}, \lambda)$ contains a singular vector $a . v_{\lambda}$ in $M(\mathfrak{g}, \lambda)_{-1}$, where a is nondegenerate.

(b) There exists a nondegenerate element $a \in \mathfrak{g}_{-1}$, such that $\lambda\left(\left[\mathfrak{g}_{1}, a\right]\right)=0$.

(c) $L(\mathfrak{g}, \lambda)$ is quasifinite.

(d) There exists a nondegenerate element $a \in \mathfrak{g}_{-1}$, such that $L(\mathfrak{g}, \lambda)$ is the irreducible quotient of the generalized Verma module $M\left(\mathfrak{g}, \mathfrak{p}^{a}, \lambda\right)$.

\section{Proof. (See Ref. 6).}

Now we will prove that $\widehat{\mathcal{D}}_{p}^{ \pm}$satisfies the properties (P1), (P2), (P3) and therefore we can apply Theorem 3.4. It is obvious that $\widehat{\mathcal{D}}_{p}^{ \pm}$satisfies (P1). In order to prove that $\widehat{\mathcal{D}}_{p}^{ \pm}$satisfies $(\mathrm{P} 2)$ and $(\mathrm{P} 3)$, we shall need the following results.

Lemma 3.5. Let $f, g, h \in \mathbb{C}[x]$ be such that $\operatorname{deg}(f g)>0$ and

$$
\left[t^{k} f(D), t^{l} g(D)\right]=t^{k+l} h(D)+\Psi\left(t^{k} f(D), t^{l} g(D)\right) C,
$$

then $\operatorname{deg}(h)=\operatorname{deg}(f)+\operatorname{deg}(g)-1$ if and only if $\operatorname{deg}(f) l \neq \operatorname{deg}(g) k$.

Proof. We suppose $f(D)=D^{i}$ and $g(D)=D^{j}$ with $i+j \neq 0$, then from (3.2)

$$
h_{i, j}(D)=(D+l)^{i} D^{j}-(D+k)^{j} D^{i}
$$

it is clear that $\operatorname{deg}\left(h_{i, j}\right) \leq i+j-1$, moreover the coefficient of $D^{i+j-1}$ is $(i l-j k)$, therefore, for this case the lemma is true. Now, let $f(x)=\sum_{i=0}^{n} f_{i} x^{i}$ and $g(x)=\sum_{j=0}^{n} g_{j} x^{j}$ be polynomials such that $n+m \neq 0$, then

$$
\left[t^{k} f(D), t^{l} g(D)\right]=\sum_{i, j} f_{i} g_{j}\left[t^{k} D^{i}, t^{l} D^{j}\right]:=\sum_{i, j} f_{i} g_{j} t^{k+l} h_{i, j}(D),
$$

with $\operatorname{deg}\left(h_{i, j}\right) \leq i+j-1$. Therefore, the proof reduces to the case $t^{k+l} h_{n, m}(D):=\left[t^{k} D^{n}, t^{l} D^{m}\right]$ that was previously considered.

Lemma 3.6. Let $\mathfrak{p}=\bigoplus_{j \in \mathbb{Z}} \mathfrak{p}_{j}$ be a $\mathbb{Z}$-graded subalgebra of $\widehat{\mathcal{D}}_{p}^{ \pm}$with $\mathfrak{p}_{0}=\left(\widehat{\mathcal{D}}_{p}^{ \pm}\right)_{0}$.

(a) If $\mathfrak{p}_{j} \neq 0$, then it has finite codimension in $\left(\widehat{\mathcal{D}}_{p}^{ \pm}\right)_{j}$.

(b) If $\mathfrak{p}_{-1} \neq 0$, then $\mathfrak{p}_{-j}$ has finite codimension in $\left(\widehat{\mathcal{D}}_{p}^{ \pm}\right)_{-j}$ for all $j \in \mathbb{N}$.

Proof. In order to prove (a), it is enough to find a family $\left\{t^{j} g_{k}(D) p(D)\right\}_{k \geqslant 1} \subset \mathfrak{p}_{j}$ with $\operatorname{deg}\left(g_{k}\right)$ $=m_{0}+2 k$ for some fixed $m_{0} \in \mathbb{Z}_{+}$(see Lemma 3.1). 
We may assume $j \neq 0$. Let $t^{j} f(D) p(D) \in \mathfrak{p}_{j}$ be nonzero. By hypothesis and Lemma 3.1, $\left(D-\frac{c}{2}\right)^{2 k+\bar{n}} p(D) \in \mathfrak{p}_{0}$ for all $k \geq 1$, then

$$
\left[t^{j} f(D) p(D),\left(D-\frac{c}{2}\right)^{2 k+\bar{n}} p(D)\right]:=t^{j} g_{k}(D) p(D) \in \mathfrak{p}_{j},
$$

and by Lemma 3.5 we obtain $\operatorname{deg}\left(g_{k}\right)=\operatorname{deg}(f)+\bar{n}+n-1+2 k$, finishing (a).

Now, in order to prove (b) we only need to see that $\mathfrak{p}_{-j} \neq 0$ for all $j \geq 1$. By induction, we suppose $\mathfrak{p}_{-j} \neq 0$ with $j \geq 1$. Then from the above argument, for all $k \geq 1$ there exists $t^{-j} g_{k}(D) p(D) \in \mathfrak{p}_{-j}$ with $\operatorname{deg}\left(g_{k}\right)=m_{0}+2 k\left(m_{0} \in \mathbb{Z}_{+}\right.$fixed) and by hypothesis there exists $t^{-1} f(D) p(D) \in \mathfrak{p}_{-1}$ that is nonzero. Hence, we can take $k_{0} \in \mathbb{N}$ such that $(n+\operatorname{deg}(f)) j \neq\left(n+m_{0}+2 k_{0}\right)$, then by Lemma 3.5 , we have that $\left[t^{-1} f(D) p(D), t^{-j} g_{k_{0}}(D) p(D)\right] \in \mathfrak{p}_{-j-1}$ is nonzero.

Corollary 3.7. (a) $\widehat{\mathcal{D}}_{p}^{ \pm}$satisfies (P2).

(b) Any parabolic subalgebra of $\widehat{\mathcal{D}}_{p}^{ \pm}$is nondegenerate.

(c) Any nonzero element of $\left(\widehat{\mathcal{D}}_{p}^{ \pm}\right)_{-1}$ is nondegenerate.

(d) $\widehat{\mathcal{D}}_{p}^{ \pm}$satisfies (P3).

Proof. Let $t^{-k} f(D) p(D) \in \widehat{\mathcal{D}}_{p}^{ \pm}$be nonzero (with $k>0$ ). Now, if we take $\operatorname{tg}(D) p(D) \in \widehat{\mathcal{D}}_{p}^{ \pm}$ with nonconstant $g$, using Lemma 3.5, we obtain that

$$
\left[t^{-k} f(D) p(D), \operatorname{tg}(D) p(D)\right] \neq 0
$$

proving that $\widehat{\mathcal{D}}_{p}^{ \pm}$satisfies (P2).

Now, let $\mathfrak{p}$ be a parabolic subalgebra of $\widehat{\mathcal{D}}_{p}^{ \pm}$, by definition there exists $j \in \mathbb{N}$ such that $\mathfrak{p}_{-j} \neq 0$ then by $(\mathrm{P} 2), \mathfrak{p}_{-1} \neq 0$, and the proof of (b) follows from Lemma 3.6(b). Finally, (c) follows from (b), and (d) follows from (c).

Let $L\left(\widehat{\mathcal{D}}_{p}^{ \pm}, \lambda\right)$ be an irreducible quasifinite highest weight module over $\widehat{\mathcal{D}}_{p}^{ \pm}$. By Theorem 3.4, there exists some monic polynomial $b\left(x-\frac{c+1}{2}\right) p(x)$ such that $\left(t^{-1} b\left(D-\frac{1+c}{2}\right) p(D)\right) v_{\lambda}=0$ (with the polynomial $b(x)$ being odd or even depending on $\widehat{\mathcal{D}}_{p}^{ \pm}$as described in Lemma 3.1). We shall call such monic polynomial of minimal degree, uniquely determined by the highest-weight $\lambda$, the characteristic polynomial of $L\left(\widehat{\mathcal{D}}_{p}^{ \pm}, \lambda\right)$.

Let us denote by $\mathbb{Z}_{+}^{(0)}$ (respectively $\mathbb{Z}_{+}^{(1)}$ ) the set all even (respectively odd) non-negative integers.

A functional $\lambda \in\left(\widehat{\mathcal{D}}_{p}^{ \pm}\right)_{0}^{*}$ is described by its labels $\triangle_{l}=-\lambda\left(\left(D-\frac{c}{2}\right)^{l} p(D)\right)$, where $l \in \mathbb{Z}_{+}^{(\bar{n})}, n=$ $\operatorname{deg}(p)$ and the central charge $\lambda(C)=\bar{c}$. We can consider the generating series

$$
\Delta_{\lambda}(x)=\sum_{l \in \mathbb{Z}_{+}^{(\bar{n})}} \frac{x^{l}}{l !} \Delta_{l} .
$$

Recall that a quasipolynomial is a linear combination of functions of the form $q(x) e^{\alpha x}$, where $q \in \mathbb{C}[x]$ and $\alpha \in \mathbb{C}$. Also we have a well-known characterization: a formal power series is a quasipolynomial (respectively even quasipolynomial) if and only if it is annihilated by the action of a nontrivial linear differential operator with constant coefficients $f(\partial)=0$, where $f(x)$ is a polynomial (respectively even polynomial). $\widehat{\mathcal{D}}_{p}^{ \pm}$.

The following theorem characterizes the irreducible quasifinite highest weight modules over

Theorem 3.8. $A \widehat{\mathcal{D}}_{p}^{ \pm}$-module $L\left(\widehat{\mathcal{D}}_{p}^{ \pm}, \lambda\right)$ is quasifinite if and only if

$$
\triangle_{\lambda}(x)=p\left(\frac{d}{d x}+\frac{c}{2}\right)\left(\frac{\phi_{\lambda}(x)}{2 \sinh \left(\frac{x}{2}\right)}\right),
$$

where $\phi_{\lambda}(x)$ is an even quasipolynomial such that $\phi_{\lambda}(0)=0$. 
Proof. Recall that $p$ satisfies

$$
p(x)=(-1)^{n} p(-x+c)
$$

where $n=\operatorname{deg}(p)$. We shall use the following identities, for $f, g \in \mathbb{C}[x]$ and $a \in \mathbb{C}$ :

$$
\begin{aligned}
f\left( \pm \frac{d}{d x}\right) e^{a x} & =f( \pm a) e^{a x} \\
e^{ \pm x\left(D-\frac{c}{2}\right)} f(D) & =f\left( \pm \frac{d}{d x}+\frac{c}{2}\right) e^{ \pm x\left(D-\frac{c}{2}\right)}, \\
e^{ \pm \frac{x}{2}} f\left(\frac{d}{d x}\right) g(x) & =f\left(\frac{d}{d x} \mp \frac{1}{2}\right) e^{ \pm \frac{x}{2}} g(x) .
\end{aligned}
$$

Using (3.5) and (3.7),

$$
\begin{aligned}
\Delta_{\lambda}(x) & =-\frac{1}{2} \lambda\left(\left(e^{x\left(D-\frac{c}{2}\right)}+(-1)^{n+1} e^{-x\left(D-\frac{c}{2}\right)}\right) p(D)\right) \\
& =-\frac{1}{2} \lambda\left(p\left(\frac{d}{d x}+\frac{c}{2}\right)\left(e^{x\left(D-\frac{c}{2}\right)}-e^{-x\left(D-\frac{c}{2}\right)}\right)\right) .
\end{aligned}
$$

Now, take $\Gamma_{\lambda}(x)$ a solution of

$$
\Delta_{\lambda}(x)=p\left(\frac{d}{d x}+\frac{c}{2}\right) \Gamma_{\lambda}(x)
$$

It follows from Theorem 3.4 that $L\left(\widehat{\mathcal{D}}_{p}^{ \pm}, \lambda\right)$ is quasifinite if and only if there exists $t^{-1} b(D-$ $\left.\frac{c+1}{2}\right) p(D) \in\left(\widehat{\mathcal{D}}_{p}^{ \pm}\right)_{-1}$ such that

$$
0=\lambda\left(\left[t\left(D-\frac{c-1}{2}\right)^{2 k+\delta} p(D), t^{-1} b\left(D-\frac{c+1}{2}\right) p(D)\right]\right)
$$

for all $k \in \mathbb{Z}$ and by Lemma 3.1 and (3.1), $b \in \mathbb{C}[x]^{(\delta)}$ where $\delta$ is given by

$$
\delta=\left\{\begin{array}{l}
\frac{\bar{n}, \quad \text { in the case } \widehat{\mathcal{D}}_{p}^{+}}{n-1,}, \text { in the case } \widehat{\mathcal{D}}_{p}^{-}
\end{array}\right.
$$

Taking generating series, (3.11) is equivalent to

$$
\begin{aligned}
0= & \frac{1}{2} \lambda\left(\left[t\left(e^{x\left(D-\frac{c-1}{2}\right)}+(-1)^{\delta} e^{-x\left(D-\frac{c-1}{2}\right)}\right) p(D), t^{-1} b\left(D-\frac{c+1}{2}\right) p(D)\right]\right) \\
= & \frac{1}{2} \lambda\left(b\left(D-\frac{c+1}{2}\right) p(D-1) p(D)\left(e^{x\left(D-\frac{c+1}{2}\right)}+(-1)^{\delta} e^{-x\left(D-\frac{c+1}{2}\right)}\right)\right. \\
& -b\left(D-\frac{c-1}{2}\right) p(D+1) p(D)\left(e^{x\left(D-\frac{c-1}{2}\right)}+(-1)^{\delta} e^{-x\left(D-\frac{c-1}{2}\right)}\right) \\
& \left.+b\left(-\frac{c+1}{2}\right) p(-1) p(0)\left(e^{-\left(\frac{c+1}{2}\right) x}+(-1)^{\delta} e^{\left(\frac{c+1}{2}\right) x}\right) C\right)
\end{aligned}
$$


Then using the identities (3.5), (3.6), (3.7), (3.8), (3.9), and (3.10)

$$
\begin{aligned}
0= & \frac{1}{2} \lambda\left(b\left(\frac{d}{d x}\right) p\left(\frac{d}{d x}+\frac{c-1}{2}\right) e^{-\frac{x}{2}} p\left(\frac{d}{d x}+\frac{c}{2}\right) e^{x\left(D-\frac{c}{2}\right)}\right. \\
& +(-1)^{\delta} b\left(-\frac{d}{d x}\right) p\left(-\frac{d}{d x}+\frac{c-1}{2}\right) e^{\frac{x}{2}} p\left(-\frac{d}{d x}+\frac{c}{2}+1\right) e^{-x\left(D-\frac{c}{2}\right)} \\
& -b\left(\frac{d}{d x}\right) p\left(\frac{d}{d x}+\frac{c+1}{2}\right) e^{\frac{x}{2}} p\left(\frac{d}{d x}+\frac{c}{2}\right) e^{x\left(D-\frac{c}{2}\right)} \\
& \left.-(-1)^{\delta} b\left(-\frac{d}{d x}\right) p\left(-\frac{d}{d x}+\frac{c+1}{2}\right) e^{-\frac{x}{2}} p\left(-\frac{d}{d x}+\frac{c}{2}-1\right) e^{-x\left(D-\frac{c}{2}\right)}\right) \\
& +\frac{1}{2}\left(b\left(\frac{d}{d x}\right) p\left(\frac{d}{d x}+\frac{c-1}{2}\right) p\left(\frac{d}{d x}+\frac{c+1}{2}\right) e^{-\left(\frac{c+1}{2}\right) x}\right. \\
& \left.+(-1)^{\delta} b\left(-\frac{d}{d x}\right) p\left(-\frac{d}{d x}+\frac{c-1}{2}\right) p\left(-\frac{d}{d x}+\frac{c+1}{2}\right) e^{\left(\frac{c+1}{2}\right) x}\right) \bar{c} \\
= & \frac{1}{2} \lambda\left(b\left(\frac{d}{d x}\right)\left[p\left(\frac{d}{d x}+\frac{c-1}{2}\right) e^{-\frac{x}{2}}-p\left(\frac{d}{d x}+\frac{c+1}{2}\right) e^{\frac{x}{2}}\right] p\left(\frac{d}{d x}+\frac{c}{2}\right) e^{x\left(D-\frac{c}{2}\right)}+\right. \\
& \left.b\left(\frac{d}{d x}\right)\left[p\left(\frac{d}{d x}+\frac{c+1}{2}\right) e^{\frac{x}{2}}-p\left(\frac{d}{d x}+\frac{c-1}{2}\right) e^{-\frac{x}{2}}\right] p\left(\frac{d}{d x}+\frac{c}{2}\right) e^{-x\left(D-\frac{c}{2}\right)}\right) \\
& +\frac{1}{2} b\left(\frac{d}{d x}\right) p\left(\frac{d}{d x}+\frac{c-1}{2}\right) p\left(\frac{d}{d x}+\frac{c+1}{2}\right)\left(e^{-\left(\frac{c+1}{2}\right) x}+e^{\left(\frac{c+1}{2}\right) x}\right) \bar{c} \\
= & b\left(\frac{d}{d x}\right)\left[p\left(\frac{d}{d x}+\frac{c+1}{2}\right) e^{\frac{x}{2}}-p\left(\frac{d}{d x}+\frac{c-1}{2}\right) e^{-\frac{x}{2}}\right] p\left(\frac{d}{d x}+\frac{c}{2}\right) \Gamma_{\lambda}(x) \\
& +b\left(\frac{d}{d x}\right) p\left(\frac{d}{d x}+\frac{c-1}{2}\right) p\left(\frac{d}{d x}+\frac{c+1}{2}\right) \cosh \left(\left(\frac{c+1}{2}\right) x\right) \bar{c} \\
= & b\left(\frac{d}{d x}\right) p\left(\frac{d}{d x}+\frac{c+1}{2}\right) p\left(\frac{d}{d x}+\frac{c-1}{2}\right)\left(2 \sinh \left(\frac{x}{2}\right) \Gamma_{\lambda}(x)+\cosh \left(\left(\frac{c+1}{2}\right) x\right) \bar{c}\right) .
\end{aligned}
$$

It follows that $L\left(\widehat{\mathcal{D}}_{p}^{ \pm}, \lambda\right)$ is quasifinite if and only if there exists $b \in \mathbb{C}[x]^{\delta}$ (see (3.12)) such that

$$
0=b\left(\frac{d}{d x}\right) p\left(\frac{d}{d x}+\frac{c+1}{2}\right) p\left(\frac{d}{d x}+\frac{c-1}{2}\right)\left(2 \sinh \left(\frac{x}{2}\right) \Gamma_{\lambda}(x)+\cosh \left(\left(\frac{c+1}{2}\right) x\right) \bar{c}\right) .
$$

Therefore, if $L\left(\widehat{\mathcal{D}}_{p}^{ \pm}, \lambda\right)$ is quasifinite, then $2 \sinh \left(\frac{x}{2}\right) \Gamma_{\lambda}(x)+\cosh \left(\left(\frac{c+1}{2}\right) x\right) \bar{c}$ is a quasipolynomial. But, using (3.9) and (3.10), we get that $\Gamma_{\lambda}(x)$ is an odd function. Hence,

$$
\phi_{\lambda}(x)=2 \sinh \left(\frac{x}{2}\right) \Gamma_{\lambda}(x)
$$

is an even quasipolynomial such that $\phi_{\lambda}(0)=0$, and using (3.10), we have

$$
\Delta_{\lambda}(x)=p\left(\frac{d}{d x}+\frac{c}{2}\right)\left(\frac{\phi_{\lambda}(x)}{2 \sinh \left(\frac{x}{2}\right)}\right) .
$$

Conversely, if (3.15) holds for some even quasipolynomial $\phi_{\lambda}$ with $\phi_{\lambda}(0)=0$, then $F(x)=\phi_{\lambda}(x)+$ $\cosh \left(\left(\frac{c+1}{2}\right) x\right) \bar{c}$ is an even quasipolynomial and it satisfies $q\left(\frac{d}{d x}\right) F(x)=0$ for some $q \in \mathbb{C}[x]^{\delta}$. In particular, we have

$$
p\left(\frac{d}{d x}+\frac{c+1}{2}\right) p\left(\frac{d}{d x}+\frac{c-1}{2}\right) q\left(\frac{d}{d x}\right) F(x)=0,
$$

and therefore $L\left(\widehat{\mathcal{D}}_{p}^{ \pm}, \lambda\right)$ is quasifinite, finishing the proof. 
The even quasipolynomial $\phi_{\lambda}(x)+\cosh \left(\left(\frac{c+1}{2}\right) x\right) \bar{c}$, where $\phi_{\lambda}(x)$ is from (3.4) and $\bar{c}$ is the central charge, can be written in the form

$$
\phi_{\lambda}(x)+\cosh \left(\left(\frac{c+1}{2}\right) x\right) \bar{c}=\sum_{i} q_{i}(x) \cosh \left(e_{i}^{+} x\right)+\sum_{j} r_{j}(x) \sinh \left(e_{j}^{-} x\right),
$$

where $q_{i}(x)$ (respectively $\left.r_{j}(x)\right)$ are nonzero even (respectively odd) polynomials and $e_{i}^{+}$(respectively $e_{j}^{-}$) are distinct complex numbers. Note that $\sum_{i} q_{i}(0)=\bar{c}$.

The expression (3.16) is unique up to a sign of $e_{i}^{+}$or a simultaneous change of signs of $e_{j}^{-}$and $r_{j}(x)$. We call $e_{i}^{+}$(respectively $e_{j}^{-}$) the even type (respectively odd type) exponents of $L\left(\widehat{\mathcal{D}}_{p}^{ \pm}, \lambda\right)$ with multiplicities $q_{i}(x)$ (respectively $r_{j}(x)$ ). We denote by $e^{+}$the set of even type exponents $e_{i}^{+}$with multiplicity $q_{i}(x)$ and by $e^{-}$the set of odd type exponents $e_{j}^{-}$with multiplicity $r_{j}(x)$. Then the pair $\left(e^{+}, e^{-}\right)$determines $L\left(\widehat{\mathcal{D}}_{p}^{ \pm}, \lambda\right)$ uniquely, and we shall also denote it as $L\left(\widehat{\mathcal{D}}_{p}^{ \pm} ; e^{+}, e^{-}\right)$.

Corollary 3.9. Let $L\left(\widehat{\mathcal{D}}_{p}^{ \pm}, \lambda\right)$ be an irreducible quasifinite highest weight module over $\widehat{\mathcal{D}}_{p}^{ \pm}$, $b\left(x-\frac{c+1}{2}\right) p(x)$ be its characteristic polynomial with $b(x) \in \mathbb{C}[x]^{\delta}$ (see $\left.(3.12)\right), \Gamma_{\lambda}(x)$ be a solution of (3.10), and let $F(x)=2 \sinh \left(\frac{x}{2}\right) \Gamma_{\lambda}(x)+\cosh \left(\left(\frac{c+1}{2}\right) x\right) \bar{c}$. Then

$$
b\left(\frac{d}{d x}\right) p\left(\frac{d}{d x}+\frac{c+1}{2}\right) p\left(\frac{d}{d x}+\frac{c-1}{2}\right) F(x)=0
$$

is the minimal order homogeneous linear differential equation with constant coefficients of the form

$$
f\left(\frac{d}{d x}\right) p\left(\frac{d}{d x}+\frac{c+1}{2}\right) p\left(\frac{d}{d x}+\frac{c-1}{2}\right) \text { with } f \in \mathbb{C}[x]^{\delta},
$$

satisfied by $F(x)$. Moreover, the exponents appearing in (3.16) are all roots of the polynomial $b(x) p\left(x+\frac{c+1}{2}\right) p\left(x+\frac{c-1}{2}\right)$.

\section{INTERPLAY BETWEEN $\widehat{\mathcal{D}}_{\boldsymbol{x}}^{\mathcal{O}, \pm}$ AND $\widehat{\mathfrak{g l}}_{\infty}^{[m]}, c_{\infty}^{[m]}, \boldsymbol{d}_{\infty}^{[m]}$, AND $\mathcal{L}_{ \pm}^{[m]}$}

Denote by $\mathrm{R}_{\mathrm{m}}$ the quotient algebra $\mathbb{C}[u] /\left(u^{m+1}\right)$ and by $\mathbf{1}$ the identity element of $\mathrm{R}_{\mathrm{m}}$. We let $\mathfrak{g}_{\infty}^{[m]}$ the Lie algebra of all matrices $\left(a_{i j}\right)_{i, j \in \mathbb{Z}}$ with finitely many nonzero diagonals with entries in $\mathrm{R}_{\mathrm{m}}$. Also denote by $E_{i j}$ the infinite matrix with 1 at $(i, j)$ place and 0 elsewhere. Letting $w t E_{i j}=j-i$ defines the principal $\mathbb{Z}$-gradation of $\mathfrak{g}_{\infty}^{[m]}$. There is a standard automorphism $v$ of $\mathfrak{g l}{ }_{\infty}^{[m]}$ given by

$$
\nu\left(E_{i, j}\right)=E_{i+1, j+1} .
$$

Consider the following two-cocycle on $\mathfrak{g l}_{\infty}^{[m]}$ with values in $\mathrm{R}_{\mathrm{m}}$ :

$$
C(A, B)=\operatorname{tr}([J, A] B),
$$

where $J=\sum_{j \leq 0} E_{j j}$, and denote by $\widehat{\mathfrak{g l}}_{\infty}^{[m]}=\mathfrak{g l}_{\infty}^{[m]} \oplus \mathrm{R}_{\mathrm{m}}$ the corresponding central extension. The $\mathbb{Z}$-gradation of this Lie algebra extends from $\mathfrak{g l}_{\infty}^{[m]}$ by letting $w t \mathrm{R}_{\mathrm{m}}=0$.

Given $\lambda \in\left(\widehat{\mathfrak{g l}}_{\infty}^{[m]}\right)_{0}^{*}$, we let

$$
\begin{aligned}
c_{j} & =\lambda\left(u^{j}\right), \\
{ }^{a} \lambda_{i}^{(j)} & =\lambda\left(u^{j} E_{i i}\right), \\
{ }^{a} h_{i}^{(j)} & ={ }^{a} \lambda_{i}^{(j)}-{ }^{a} \lambda_{i+1}^{(j)}+\delta_{i, 0} c_{j},
\end{aligned}
$$

where $i \in \mathbb{Z}$ and $j=0, \ldots, m$. The superscript $a$ corresponds to the type A Lie algebra $\widehat{\mathfrak{g l}}_{\infty}^{[m]}$. Let $L\left(\left.\widehat{\mathfrak{g}}\right|_{\infty} ^{[m]}, \lambda\right)$ be the irreducible highest weight $\widehat{\mathfrak{g l}}_{\infty}^{[m]}$-module with highest weight $\lambda$. The ${ }^{a} \lambda_{i}^{(j)}$ are called the labels and $c_{j}$ are the central charges of $L\left(\widehat{\mathfrak{g l}}_{\infty}^{[m]}, \lambda\right)$. 
Consider the vector space $\mathrm{R}_{\mathrm{m}}\left[t, t^{-1}\right]$ and take its basis $v_{i}=t^{i}(i \in \mathbb{Z})$ over $\mathrm{R}_{\mathrm{m}}$. Let us consider the following $\mathbb{C}$-bilinear forms on this space:

$$
\begin{aligned}
& C\left(u^{m} v_{i}, u^{n} v_{j}\right)=u^{m}(-u)^{n}(-1)^{i} \delta_{i, 1-j}, \\
& D\left(u^{m} v_{i}, u^{n} v_{j}\right)=u^{m}(-u)^{n} \delta_{i, 1-j} .
\end{aligned}
$$

Denote by $\bar{c}_{\infty}^{[m]}$ and $\bar{d}_{\infty}^{[m]}$ the Lie subalgebras of $\mathfrak{g l}_{\infty}^{[m]}$ which preserve the bilinear forms $C$ and $D$, respectively. We have,

$$
\begin{aligned}
& \bar{c}_{\infty}^{[m]}=\left\{A \in \mathfrak{g l}_{\infty}^{[m]} \mid A_{i, j}(u)=(-1)^{i+j+1} A_{1-j, 1-i}(-u)\right\}, \\
& \bar{d}_{\infty}^{[m]}=\left\{A \in \mathfrak{g l}_{\infty}^{[m]} \mid A_{i, j}(u)=-A_{1-j, 1-i}(-u)\right\} .
\end{aligned}
$$

Denote by $c_{\infty}^{[m]}=\bar{c}_{\infty}^{[m]} \oplus \mathrm{R}_{\mathrm{m}}$ and $d_{\infty}^{[m]}=\bar{d}_{\infty}^{[m]} \oplus R_{m}$ the central extension of $\bar{c}_{\infty}^{[m]}$ and $\bar{d}_{\infty}^{[m]}$, respectively, given by the restriction of the two-cocycle (4.2). These subalgebras inherit from $\widehat{\mathfrak{g l}}_{\infty}^{[m]}$ the principal $\mathbb{Z}$-gradation.

Let $\mathfrak{g}$ stand for $c_{\infty}^{[m]}$ or $d_{\infty}^{[m]}$. Given $\lambda \in(\mathfrak{g})_{0}^{*}$, denote by $L(\mathfrak{g}, \lambda)$ the irreducible highest weight module over $\mathfrak{g}$ with highest weight $\lambda$. We let

$$
\begin{aligned}
c_{j} & =\lambda\left(u^{j}\right), \\
{ }^{g} \lambda_{i}^{(j)} & =\lambda\left(u^{j} E_{i i}-(-u)^{j} E_{1-i, 1-i}\right), \\
{ }^{g} h_{i}^{(j)} & ={ }^{\mathfrak{g}} \lambda_{i}^{(j)}-{ }^{\mathfrak{g}} \lambda_{i+1}^{(j)}, \\
{ }^{g} h_{0}^{(j)} & ={ }^{\mathfrak{g}} \lambda_{1}^{(j)}+c_{j} \quad(j \text { even }),
\end{aligned}
$$

where $i \in \mathbb{Z}, j=0, \ldots, m$ and the superscript $g$ represents $c$ or $d$ depending on whether $\mathfrak{g}$ is $c_{\infty}^{[m]}$ or $d_{\infty}^{[m]}$. The ${ }^{g} \lambda_{i}^{(j)}$ are called the labels and $c_{j}$ are the central charges of $L(\mathfrak{g}, \lambda)$.

We define

$$
\begin{aligned}
\overline{\mathcal{L}}_{ \pm}^{[m]}= & \left\{A \in \mathfrak{g} \mathfrak{g}_{\infty}^{[m]} \mid A_{i, j}(u)=-(\mp 1)^{i+j} A_{-j,-i}(-u) \text { if } i j>0 \vee i=j=0 ;\right. \\
& A_{i, j}(u)=(\mp 1)^{i+j} A_{-j,-i}(-u) \text { if } i j<0 ; A_{-i, 0}(u)=(\mp 1)^{i} u A_{0, i}(-u) ; \\
& \left.A_{i, 0}(u)=-(\mp 1)^{i} u A_{0,-i}(-u) \text { for } i \in \mathbb{N}\right\},
\end{aligned}
$$

subalgebras of $\mathfrak{g} \mathfrak{l}_{\infty}^{[m]}$. Denote by $\mathcal{L}_{ \pm}^{[m]}=\overline{\mathcal{L}}_{ \pm}^{[m]} \oplus \mathrm{R}_{\mathrm{m}}$ the central extension of $\overline{\mathcal{L}}_{ \pm}^{[m]}$, given by the restriction of the two-cocycle (4.2). These subalgebras inherit from $\widehat{\mathfrak{g l}}_{\infty}^{[\mathrm{m}]}$ the principal $\mathbb{Z}$-gradation.

Given $\lambda \in\left(\mathcal{L}_{ \pm}^{[m]}\right)_{0}^{*}$, we let

$$
\begin{aligned}
c_{j} & =\lambda\left(u^{j}\right), \\
{ }^{ \pm} \lambda_{i}^{(j)} & =\lambda\left(u^{j} E_{i, i}-(-u)^{j} E_{-i,-i}\right), \\
{ }^{ \pm} h_{i}^{(j)} & ={ }^{ \pm} \lambda_{i}^{(j)}-{ }^{ \pm} \lambda_{i+1}^{(j)}+\delta_{i, 0} c_{j},
\end{aligned}
$$

where $i \in \mathbb{Z}$ and $j=0, \ldots, m$. The superscript \pm corresponds to the Lie algebras $\mathcal{L}_{ \pm}^{[m]}$. Let $L\left(\mathcal{L}_{ \pm}^{[m]}, \lambda\right)$ be the irreducible highest weight $\widehat{\mathfrak{g l}}_{\infty}^{[m]}$-module with highest weight $\lambda$. The ${ }^{ \pm} \lambda_{i}^{(j)}$ are called the labels and $c_{j}$ are the central charges of $L\left(\mathcal{L}_{ \pm}^{[m]}, \lambda\right)$.

Let $\mathcal{O}$ denote the algebra of all holomorphic function on $\mathbb{C}$ with the topology of uniform convergence on compact sets and $\mathcal{O}^{(1)}$ (respectively $\mathcal{O}^{(0)}$ ) the set of odd (respectively even) holomorphic function. We consider the vector space $\mathcal{D}^{\mathcal{O}}$ spanned by the differential operators (of infinite order) of the form $t^{k} f(D)$, where $f \in \mathcal{O}$. The bracket in $\mathcal{D}$ naturally extends to $\mathcal{D}^{\mathcal{O}}$. Similarly, we define a completion $\mathcal{D}_{x}^{\mathcal{O},-}$ (respectively $\mathcal{D}_{x}^{\mathcal{O},+}$ ) of $\mathcal{D}_{x}^{-}$(respectively $\mathcal{D}_{x}^{+}$) consisting of all differential operators of the form $t^{k} f\left(D+\frac{k}{2}\right) D$ where $f \in \mathcal{O}^{(\bar{k})}$ (respectively $f \in \mathcal{O}^{(0)}$ ).

Then the two-cocycle $\Psi$ on $\mathcal{D}$ (respectively $\mathcal{D}_{x}^{ \pm}$) extends to a two-cocycle on $\mathcal{D}^{\mathcal{O}}$ (respectively $\mathcal{D}_{x}^{\mathcal{O}, \pm}$ ). We denote the corresponding central extension by $\widehat{\mathcal{D}}^{\mathcal{O}}=\mathcal{D}^{\mathcal{O}} \oplus \mathbb{C} \mathbf{C}$ (respectively $\widehat{\mathcal{D}}_{x}^{\mathcal{O}, \pm}=$ $\left.\mathcal{D}_{x}^{\mathcal{O}, \pm} \oplus \mathbf{C} \mathbf{C}\right)$. 
Given $s \in \mathbb{C}$, we will consider a family of homomorphisms of Lie algebras $\varphi_{s}^{[m], \pm}: \mathcal{D}_{x}^{\mathcal{O}, \pm} \rightarrow$ $\mathfrak{g l}_{\infty}^{[m]}$ defined by

$$
\begin{aligned}
\varphi_{s}^{[m], \pm}\left(t^{k} f\left(D+\frac{k}{2}\right) D\right) & =\sum_{j \in \mathbb{Z}} f\left(-j+\frac{k}{2}+s+u\right)(-j+s+u) E_{j-k, j} \\
& =\sum_{j \in \mathbb{Z}} \sum_{i=0}^{m} \frac{(f(-j+k / 2+s)(-j+s))^{(i)}}{i !} u^{i} E_{j-k, j} \\
& =\sum_{j \in \mathbb{Z}} \sum_{i=0}^{m} \frac{f^{(i)}\left(-j+\frac{k}{2}+s\right)}{i !}((-j+s)+u) u^{i} E_{j-k, j},
\end{aligned}
$$

where $f^{(i)}$ denotes the $i$ th derivative. Note that $\varphi_{s}^{[m],{ }^{ \pm}}$is the restriction to $\mathcal{D}_{x}^{\mathcal{O}, \pm}$ of the homomorphism (3.2.1) in Ref. 8.

Remark 4.1. The principal $\mathbb{Z}$-gradations on $\mathcal{D}_{x}^{\mathcal{O}, \pm}$ and $\mathfrak{g l}_{\infty}^{[m]}$ are compatible under the homomorphisms $\varphi_{s}^{[m], \pm}$.

Let

$$
\begin{aligned}
& I_{s, k}^{[m],-}=\left\{f \in \mathcal{O}^{(\bar{k})}: f^{(i)}(-j+k / 2+s)=0 \text { for all } j \in \mathbb{Z}, 0 \leqslant i \leqslant m\right\}, \\
& I_{s, k}^{[m],+}=\left\{f \in \mathcal{O}^{(0)}: f^{(i)}(-j+k / 2+s)=0 \text { for all } j \in \mathbb{Z}, 0 \leqslant i \leqslant m\right\},
\end{aligned}
$$

and let

$$
\begin{aligned}
& J_{s}^{[m],-}=\bigoplus_{k \in \mathbb{Z}}\left\{t^{k} f(D+k / 2): f \in I_{s, k}^{[m],-}\right\}, \\
& J_{s}^{[m],+}=\bigoplus_{k \in \mathbb{Z}}\left\{t^{k} f(D+k / 2): f \in I_{s, k}^{[m],+}\right\} .
\end{aligned}
$$

Proposition 4.2. Given $s \in(\mathbb{C}-\mathbb{Z} / 2)$ and $m \in \mathbb{Z}_{+}$we have the following exact sequence of Lie algebras:

$$
0 \rightarrow J_{s}^{[m], \pm} \rightarrow \mathcal{D}_{x}^{\mathcal{O}, \pm} \stackrel{\varphi_{s}^{[m], \pm}}{\longrightarrow} \mathfrak{g l}_{\infty}^{[m]} \rightarrow 0
$$

Proof. It is clear that $\operatorname{ker} \varphi_{s}^{[m], \pm}=J_{s}^{[m], \pm}$. We only need to prove that $\varphi_{s}^{[m], \pm}$ is surjective. We recall the following well-known fact: for every discrete sequence of points in $\mathbb{C}$ and a non-negative integer $m$ there exists $a(x) \in \mathcal{O}$ having the prescribed values of its first $m$ derivatives at these points.

Case $\varphi_{s}^{[m],-}$ (respectively $\varphi_{s}^{[m],+}$ ): since $s \notin \mathbb{Z} / 2$ the sequence $\{-j+k / 2+s\}_{j \in \mathbb{Z}}$ and $\{j-$ $k / 2-s\}_{j \in \mathbb{Z}}$ are disjoint. We fix $0 \leq i_{0} \leq m$ and $j_{0}, k \in \mathbb{Z}$, then there exists $a(x) \in \mathcal{O}$ such that

- if $k$ is even, $a^{(i)}(-j+k / 2+s)=a^{(i)}(j-k / 2-s)=\delta_{i, i_{0}} \delta_{j, j_{0}} / 2$,

- if $k$ is odd, $a^{(i)}(-j+k / 2+s)=2 \delta_{i, i_{0}} \delta_{j, j_{0}}, a^{(i)}(j-k / 2-s)=\delta_{i, i_{0}} \delta_{j, j_{0}}$

$\left(\right.$ respectively $\left.a^{(i)}(-j+k / 2+s)=a^{(i)}(j-k / 2-s)=\delta_{i, i_{0}} \delta_{j, j_{0}} / 2\right)$, and let

$$
g(x)=i_{0} !\left(a(x)+(-1)^{k} a(-x)\right)
$$

(respectively $\left.g(x)=i_{0} !(a(x)+a(-x))\right)$. Then

$$
\varphi_{s}^{[m], \pm}\left(t^{k} g(D+k / 2) D\right)=\left(u+\left(s-j_{0}\right)\right) u^{i_{0}} E_{j_{0}-k, j_{0}},
$$

and since $\left\{\left(u+\left(s-j_{0}\right)\right) u^{i}\right\}_{i=0}^{m}$ is a basis of $\mathbf{R}_{\mathrm{m}}$, then $\varphi_{s}^{[m], \pm}$ is surjective, finishing the proof. 
Proposition 4.3. For $s=\frac{1}{2}$, we have the following exact sequence of Lie algebras:

$$
0 \rightarrow J_{\frac{1}{2}}^{[m]} \rightarrow \mathcal{D}_{x}^{\mathcal{O}, \pm} \rightarrow \overline{\mathfrak{g}}_{ \pm} \rightarrow 0,
$$

where $\overline{\mathfrak{g}}_{+}:=\bar{c}_{\infty}^{[m]}$ and $\overline{\mathfrak{g}}_{-}:=\bar{d}_{\infty}^{[m]}$.

Proof. The homomorphism $\varphi_{\frac{1}{2}}^{[m]}: \mathcal{D}_{x}^{\mathcal{O}} \rightarrow \mathfrak{g l}_{\infty}^{[m]}$ defined by

$$
\begin{aligned}
\varphi_{\frac{1}{2}}^{[m]}\left(t^{k} f(D) D\right) & =\sum_{j \in \mathbb{Z}} f\left(u+\frac{1}{2}-j\right)\left(u+\frac{1}{2}-j\right) E_{j-k, j} \\
& =\sum_{j \in \mathbb{Z}} \sum_{i=0}^{m} \frac{f^{(i)}\left(\frac{1}{2}-j\right)}{i !}\left(\left(\frac{1}{2}-j\right)+u\right) u^{i} E_{j-k, j}
\end{aligned}
$$

is surjective and the anti-involution $\sigma_{ \pm}$is transferred through this homomorphism to an antiinvolution $w_{ \pm}$in $\mathfrak{g l}_{\infty}^{[m]}$ that satisfies

$$
w_{ \pm}\left(\left(u+\frac{1}{2}-j\right) f(u) E_{i, j}\right)=( \pm 1)^{i+j}\left(-u+\frac{1}{2}-i\right) f(-u) E_{1-j, 1-i},
$$

with $f \in \mathbb{C}[x]$, from which it is easy to see that

$$
w_{ \pm}\left(f(u) E_{i, j}\right)=( \pm 1)^{i+j}\left(-u+\frac{1}{2}-i\right)\left(-u+\frac{1}{2}-j\right)^{-1} f(-u) E_{1-j, 1-i} .
$$

Then, the Lie algebra of $-\sigma_{ \pm}$-fixed points in $\mathcal{D}_{x}^{\mathcal{O}}$ (namely $\mathcal{D}_{x}^{\mathcal{O}, \pm}$ ) maps surjectively to the Lie algebra of $-w_{ \pm}$-fixed points in $\mathfrak{g}_{\infty}^{[m]}$.

Now, we define the automorphism $T: \mathfrak{g l}_{\infty}^{[m]} \longrightarrow \mathfrak{g l}_{\infty}^{[m]}$ by

$$
\begin{aligned}
T\left(u^{l} E_{i, j}\right) & =\prod_{k=i}^{j-1}\left(u-\left(k+\frac{1}{2}\right)\right) u^{l} E_{i, j} \quad \text { if } i<j, \\
T\left(u^{l} E_{i, i}\right) & =u^{l} E_{i, i}, \\
T\left(u^{l} E_{i, j}\right) & =\prod_{k=j}^{i-1}\left(u-\left(k+\frac{1}{2}\right)\right)^{-1} u^{l} E_{i, j} \quad \text { if } i>j .
\end{aligned}
$$

On the other hand, let $\rho_{ \pm}\left(f(u) E_{i, j}\right)=(\mp 1)^{i+j} f(-u) E_{1-j, 1-i}$ be the anti-involution in $\mathfrak{g l}_{\infty}^{[m]}$ that define $\overline{\mathfrak{g}}_{ \pm}$, then using (4.7) we have that

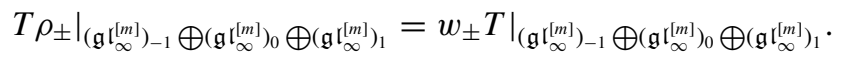

Then, since $\mathfrak{g l}_{\infty}^{[m]}$ is generated by $\left(\mathfrak{g l}_{\infty}^{[m]}\right)_{-1} \bigoplus\left(\mathfrak{g l}_{\infty}^{[m]}\right)_{0} \bigoplus\left(\mathfrak{g l}_{\infty}^{[m]}\right)_{1}$ and using (4.8), we obtain that $\rho_{ \pm}=T^{-1} w_{ \pm} T$. As before, the Lie algebra of $-w_{ \pm}$-fixed points in $\mathfrak{g}_{\infty}^{[m]}$ maps surjectively via $T^{-1}$ to the Lie algebra of $-\rho_{ \pm}$-fixed points in $\mathfrak{g g}_{\infty}^{[m]}$, namely $\overline{\mathfrak{g}}_{ \pm}$. So $T^{-1} \varphi_{\frac{1}{2}}^{[m]}$ maps surjectively $\mathcal{D}_{x}^{\mathcal{O}, \pm}$ in $\overline{\mathfrak{g}}_{ \pm}$and since $T^{-1}$ is an automorphism it is clear that $\operatorname{ker} T^{-1} \varphi_{\frac{1}{2}}^{[m], \pm}=J_{\frac{1}{2}}^{[m], \pm}$, finishing the proof.

Proposition 4.4. For $s=0$, we have the following exact sequence of Lie algebras:

$$
0 \rightarrow J_{0}^{[m]} \rightarrow \mathcal{D}_{x}^{\mathcal{O}, \pm} \rightarrow \overline{\mathcal{L}}_{ \pm}^{[m]} \rightarrow 0 .
$$


Proof. We consider the following morphisms of Lie algebras: $\varphi_{0}^{[m]}$ as in (4.6) and $T: \mathfrak{g}_{\infty}^{[m]} \longrightarrow$ $\mathfrak{g}_{\infty}^{[m]}$ defined by

$$
\begin{aligned}
T\left(u^{l} E_{i, j}\right) & =\prod_{\substack{k=i \\
k \neq 0}}^{j-1}(u-k) u^{l} E_{i, j} \quad \text { if } i<j, \\
T\left(u^{l} E_{i, i}\right) & =u^{l} E_{i, i}, \\
T\left(u^{l} E_{i, j}\right) & =\prod_{\substack{k=i \\
k \neq 0}}^{i-1}(u-k)^{-1} u^{l} E_{i, j} \quad \text { if } i>j,
\end{aligned}
$$

note that $T\left(u^{l} E_{i, j}\right)=u^{l} T\left(E_{i, j}\right)$. Then, $T \varphi_{0}^{[m]}: \mathcal{D}_{x}^{\mathcal{O}, \pm} \rightarrow \overline{\mathcal{L}}_{ \pm}^{[m]}$ is surjective and since $T$ is an automorphism, it is clear that $\operatorname{ker} T \varphi_{0}^{[m]}=J_{0}^{[m], \pm}$, finishing the proof.

Remark 4.5. (a) For $s=0$ and $s=\frac{1}{2}$, by an abuse the notation we will denote again $\varphi_{s}^{[m], \pm}$ the surjective homomorphism of Propositions 4.3 and 4.4.

(b) For $s \in \mathbb{Z}$ (respectively $s \in \mathbb{Z}+\frac{1}{2}$ ) the image of $\mathcal{D}_{x}^{\mathcal{O}, \pm}$ under the homomorphism $\varphi_{s}^{[m], \pm}$ is $v^{\widetilde{s}} \varphi_{0}^{[m], \pm}\left(\mathcal{D}_{x}^{\mathcal{O}, \pm}\right)\left(\right.$ respectively $v^{\widetilde{s}} \varphi_{\frac{1}{2}}^{[m], \pm}\left(\mathcal{D}_{x}^{\mathcal{O}, \pm}\right)$ ), where $v$ was defined in $(4.1)$ and $\widetilde{s}=s$ (respectively $\left.\widetilde{s}=s-\frac{1}{2}\right)$. Therefore, we will only consider $s=0, \frac{1}{2}$ throughout the article.

(c) Observe that Proposition 4.4 is the corrected version of Proposition 5.3 in Ref. 4.

Now we want to extend the homomorphism $\varphi_{s}^{[m], \pm}$ to a homomorphism between the central extensions of the corresponding Lie algebras. Define

$$
\eta_{i}(x, s)=\frac{e^{(s-1 / 2) x}+(-1)^{i} e^{-(s-1 / 2) x}}{2} \frac{x^{i}}{i !} \quad\left(i \in \mathbb{Z}_{+}, s \in \mathbb{C}\right) .
$$

The functions $\eta_{i}(x, s)$ satisfy

$$
\begin{aligned}
\eta_{i}(x,-s) & =(-1)^{i} \eta_{i}(x, s+1), \\
\eta_{0}(x, s+1 / 2) & =\cosh (s x) .
\end{aligned}
$$

Proposition 4.6. The homomorphism $\varphi_{s}^{[m], \pm}$ lifts to a Lie algebra homomorphism $\widehat{\varphi}_{s}^{[m], \pm}$ of the corresponding central extensions as follows:

$$
\begin{aligned}
\left.\widehat{\varphi}_{s}^{[m], \pm}\right|_{\left(\widehat{\mathcal{D}}_{x}^{ \pm}\right)_{j}}= & \left.\varphi_{s}^{[m], \pm}\right|_{\left(\mathcal{D}_{x}^{ \pm}\right)_{j}}(\text { for } j \neq 0), \\
\widehat{\varphi}_{s}^{[m], \pm}(\sinh (x D))= & \frac{1}{2} \sum_{i=0}^{m} \sum_{j \in \mathbb{Z}} \frac{\eta_{i}(x, s-j+1)-\eta_{i}(x, s-j)}{\sinh (x / 2)} u^{i} E_{j, j} \\
& -\frac{1}{2} \sum_{i=0}^{m} \frac{\eta_{i}(x, s)}{\sinh (x / 2)} u^{i} c_{0}+\frac{1}{2} \frac{\cosh (x / 2)}{\sinh (x / 2)} c_{0}, \\
\widehat{\varphi}_{s}^{[m], \pm}(C)= & 1 .
\end{aligned}
$$

Proof. Note that $\left(\widehat{\mathcal{D}}_{x}^{+}\right)_{0}=\left(\widehat{\mathcal{D}}_{x}^{-}\right)_{0}$ and therefore $\left.\widehat{\varphi}_{s}^{[m],+}\right|_{\left(\widehat{\mathcal{D}}_{x}^{+}\right)_{0}}=\left.\widehat{\varphi}_{s}^{[m],-}\right|_{\left(\widehat{\mathcal{D}}_{x}^{-}\right)_{0}}$. See Proposition 5.2 in Ref. 4. 
Let $\bar{m}=\left(m_{1}, \ldots, m_{N}\right) \in \mathbb{Z}_{+}^{N}$ and $\bar{s}=\left(s_{1}, \ldots, s_{N}\right) \in \mathbb{C}^{N}$ be such that $s_{i} \in \mathbb{Z}$ implies $s_{i}=0$, $s_{i} \in \mathbb{Z}+\frac{1}{2}$ implies $s_{i}=\frac{1}{2}$ and $s_{i} \neq \pm s_{j} \bmod \mathbb{Z}$ for $i \neq j$, combining Propositions 4.2, 4.3, 4.4, and 4.6 we obtain the following result.

Proposition 4.7. Given $\bar{m}$ and $\bar{s}$ as above, we have the following exact sequence of Lie algebras:

$$
0 \rightarrow \bigcap_{i=1}^{N} J_{s_{i}}^{\left[m_{i}\right], \pm} \rightarrow \widehat{\mathcal{D}}_{x}^{\mathcal{O}, \pm} \stackrel{\hat{\varphi}_{s}^{[m], \pm}}{\longrightarrow} \mathfrak{g}_{ \pm}^{[\bar{m}]} \rightarrow 0
$$

where $\widehat{\varphi}_{\bar{s}}^{[\bar{m}], \pm}=\bigoplus_{i=0}^{N} \widehat{\varphi}_{s_{i}}^{\left[m_{i}\right], \pm}$ and $\mathfrak{g}_{ \pm}^{[\bar{m}]}=\bigoplus_{i=0}^{N} \mathfrak{g}_{ \pm}^{\left[m_{i}\right]}$ with

$$
\mathfrak{g}_{+}^{\left[m_{i}\right]}= \begin{cases}\widehat{\mathfrak{g l}}_{\infty}^{\left[m_{i}\right]}, & \text { if } s_{i} \neq 0, \frac{1}{2} \\ c_{\infty}^{\left[m_{i}\right]}, & \text { if } s_{i}=\frac{1}{2} \\ \mathcal{L}_{+}^{\left[m_{i}\right]}, & \text { if } s_{i}=0\end{cases}
$$

and

$$
\mathfrak{g}_{-}^{\left[m_{i}\right]}=\left\{\begin{array}{ll}
\widehat{\mathfrak{g l}}_{\infty}^{\left[m_{i}\right]}, & \text { if } s_{i} \neq 0, \frac{1}{2} \\
d_{\infty}^{[m]}, & \text { if } s_{i}=\frac{1}{2} \\
\mathcal{L}_{-}^{\left[m_{i}\right]}, & \text { if } s_{i}=0
\end{array} .\right.
$$

\section{REALIZATION OF QUASIFINITE HEIGHT MODULES OF $\widehat{\mathcal{D}}_{x}^{\mathcal{O}, \pm}$}

Let $\mathfrak{g}^{[m]}$ stand for $\widehat{\mathfrak{g l}}_{\infty}^{[m]}$ or $c_{\infty}^{[m]}$ or $d_{\infty}^{[m]}$ or $\mathcal{L}_{ \pm}^{[m]}$. The proof of the following proposition is standard.

Proposition 5.1. The $\mathfrak{g}^{[m]}$-module $L\left(\mathfrak{g}^{[m]}, \lambda\right)$ is quasifinite if and only if all but finitely many of the ${ }^{*} h_{k}^{(i)}$ are zero, where * represents a or c or d or \pm depending $\mathfrak{g}^{[m]}$ is $\widehat{\mathfrak{g l}}_{\infty}^{[m]}$ or $c_{\infty}^{[m]}$ or $d_{\infty}^{[m]}$ or $\mathcal{L}_{ \pm}^{[m]}$.

Let $\bar{m}=\left(m_{1}, \ldots, m_{N}\right) \in \mathbb{Z}_{+}^{N}$ and $\bar{s}=\left(s_{1}, \ldots, s_{N}\right) \in \mathbb{C}^{N}$ be such that $s_{i} \in \mathbb{Z}$ implies $s_{i}=0$, $s_{i} \in \mathbb{Z}+\frac{1}{2}$ implies $s_{i}=\frac{1}{2}$ and $s_{i} \neq \pm s_{j} \bmod \mathbb{Z}$ for $i \neq j$, take a quasifinite $\lambda_{i} \in\left(\mathfrak{g}_{ \pm}^{\left[m_{i}\right]}\right)_{0}^{*}$ for each $i=$ $1, \ldots, N$ and let $L\left(\mathfrak{g}_{ \pm}^{\left[m_{i}\right]}, \lambda_{i}\right)$ be the corresponding irreducible $\mathfrak{g}_{ \pm}^{\left[m_{i}\right]}$-module. Let $\bar{\lambda}=\left(\lambda_{1}, \ldots, \lambda_{N}\right)$. Then the tensor product

$$
L\left(\mathfrak{g}_{ \pm}^{[\bar{m}]}, \bar{\lambda}\right)=\bigotimes_{i=1}^{N} L\left(\mathfrak{g}_{ \pm}^{\left[m_{i}\right]}, \lambda_{i}\right)
$$

is an irreducible $\mathfrak{g}_{ \pm}^{[\bar{m}]}$-module, with $\mathfrak{g}_{ \pm}^{[\bar{m}]}=\bigoplus_{i=1}^{N} \mathfrak{g}_{ \pm}^{\left[m_{i}\right]}$ as in Proposition 4.7. The module $L\left(\mathfrak{g}^{[\bar{m}]}, \bar{\lambda}\right)$ can be regarded as a $\widehat{\mathcal{D}}_{x}^{ \pm}$-module via the homomorphism $\widehat{\varphi}_{\bar{s}}^{\bar{m}], \pm}$, and will be denoted by $L_{\bar{s}}^{[\bar{m}]}(\bar{\lambda})$. We shall need the following proposition. Its proof is analogous to that of Proposition 4.3. ${ }^{8}$

Proposition 5.2. Let $V$ be a quasifinite $\widehat{\mathcal{D}}_{x}^{ \pm}$-module. Then the action of $\widehat{\mathcal{D}}_{x}^{ \pm}$on $V$ naturally extends to the action of $\left(\widehat{\mathcal{D}}_{x}^{\mathcal{O}, \pm}\right)_{k}$ on $V$ for any $k \neq 0$.

Theorem 5.3. Let $V$ be a quasifinite $\mathfrak{g}_{ \pm}^{[\bar{m}]}$-module, which is regarded as a $\widehat{\mathcal{D}}_{x}^{ \pm}$-module via the homomorphism $\widehat{\varphi}_{\bar{s}}^{[\bar{m}], \pm}$. Then any $\widehat{\mathcal{D}}_{x}^{ \pm}$-submodule of $V$ is also a $\mathfrak{g}_{ \pm}^{[\bar{m}]}$-submodule. In particular, the $\widehat{\mathcal{D}}_{x}^{ \pm}$-modules $L_{\bar{s}}^{[\bar{m}]}(\bar{\lambda})$ are irreducible if $\bar{s}=\left(s_{1}, \ldots, s_{N}\right) \in \mathbb{C}^{N}$ is such that $s_{i} \in \mathbb{Z}$ implies $s_{i}=$ $0, s_{i} \in \mathbb{Z}+\frac{1}{2}$ implies $s_{i}=\frac{1}{2}$, and $s_{i} \neq \pm s_{j} \bmod \mathbb{Z}$ for $i \neq j$.

Proof. Let $U$ be a $\widehat{\mathcal{D}}_{x}^{ \pm}$-submodule of $V$, then $U$ is a quasifinite $\widehat{\mathcal{D}}_{x}^{ \pm}$-module as well, hence by Proposition 5.2 it can be extended to $\left(\widehat{\mathcal{D}}_{x}^{\mathcal{O}, \pm}\right)_{k}$ for any $k \neq 0$. By Proposition 4.7, the map $\widehat{\varphi}_{\bar{s}}^{[\bar{m}], \pm}:\left(\widehat{\mathcal{D}}_{x}^{\mathcal{O}, \pm}\right)_{k} \rightarrow\left(\mathfrak{g}_{ \pm}^{[\bar{m}]}\right)_{k}$ is surjective for any $k \neq 0$. Therefore, $U$ is invariant with respect to all 
graded subspaces $\left(\mathfrak{g}_{ \pm}^{[\bar{m}]}\right)_{k}(k \neq 0)$ of $\mathfrak{g}_{ \pm}^{[\bar{m}]}$. Using that $\mathfrak{g}_{ \pm}^{[\bar{m}]}$ coincides with its derived algebra, we finish the proof.

Given an irreducible highest weight $\widehat{\mathcal{D}}_{x}^{ \pm}$-module $L\left(\widehat{\mathcal{D}}_{x}^{ \pm}, \lambda\right)$, using Theorem 3.8, we have that it is quasifinite if and only if

$$
\Delta_{\lambda}(x)=\frac{d}{d x}\left(\frac{\phi_{\lambda}(x)}{2 \sinh \left(\frac{x}{2}\right)}\right),
$$

where $\phi_{\lambda}(x)$ is an even quasipolynomial such that $\phi_{\lambda}(0)=0$.

On the other hand, observe that a functional $\lambda \in\left(\widehat{\mathcal{D}}_{x}^{ \pm}\right)_{0}^{*}$ is also characterized by $\Gamma_{l}=-\lambda\left(D^{l+1}\right)$, where $l \in \mathbb{Z}_{+}^{0}$, and the central charge $\lambda(C)=\bar{c}$, cf. (3.3). Consider the new generating series:

$$
\Gamma_{\lambda}(x)=\sum_{l \in \mathbb{Z}_{+}^{0}} \frac{x^{l+1}}{(l+1) !} \Gamma_{l}=-\lambda(\sinh (x D)),
$$

observe that $\Gamma_{\lambda}(x)$ satisfies (3.10), then using (3.14) we obtain

$$
\Gamma_{\lambda}(x)=\frac{\phi_{\lambda}(x)}{2 \sinh \left(\frac{x}{2}\right)} .
$$

We will show that in fact all the quasifinite $\widehat{\mathcal{D}}_{x}^{ \pm}$-module can be realized as some $L_{\bar{s}}^{[\bar{m}]}\left(\mathfrak{g}_{ \pm}^{[\bar{m}]}, \lambda\right)$, and this is done by the study of exponents and multiplicities using the computation of the generating series $\Gamma_{m, s, \lambda}(x)$ of the highest weight $\widehat{\mathcal{D}}_{x}^{\mathcal{O}, \pm}$-module $L_{s}^{[m]}\left(\mathfrak{g}_{ \pm}^{[m]}, \lambda\right)$.

Proposition 5.4. For $s \in(\mathbb{C}-\mathbb{Z} / 2)$, consider the embedding $\widehat{\varphi}_{s}^{[m]}: \widehat{\mathcal{D}}_{x}^{ \pm} \rightarrow \widehat{\mathfrak{g l}}_{\infty}^{[m]}$. Then the $\widehat{\mathfrak{g l}}_{\infty}^{[m]}$ module $L\left(\widehat{\mathfrak{g l}}_{\infty}^{[m]}, \lambda\right)$ regarded as a $\widehat{\mathcal{D}}_{x}^{ \pm}$-module via $\widehat{\varphi}_{s}^{[m]}$ is isomorphic to $L\left(\widehat{\mathcal{D}}_{x}^{ \pm} ; e^{+}, e^{-}\right)$where $e^{+}, e^{-}$ consist of exponents $\left(s-j-\frac{1}{2}\right)$ with $j \in \mathbb{Z}$ and multiplicities

$$
\sum_{\substack{0 \leqslant i \leqslant m, i \text { even }}} \frac{{ }^{a} h_{j}^{(i)} x^{i}}{i !} \text { and } \sum_{\substack{0 \leqslant i \leqslant m, i \text { odd }}} \frac{{ }^{a} h_{j}^{(i)} x^{i}}{i !},
$$

respectively.

Proof. By Proposition 5.1 and Theorem 5.3, the $\widehat{\mathcal{D}}_{x}^{ \pm}$-module $L\left(\widehat{\mathfrak{g l}}_{\infty}^{[m]}, \lambda\right)$ is an irreducible quasifinite highest weight module. Using (4.10), the central charge $c=\bar{c}$. Using the explicit expression of the homomorphism $\widehat{\varphi}_{s}^{[m]}: \widehat{\mathcal{D}}_{x}^{ \pm} \rightarrow \widehat{\mathfrak{g l}}_{\infty}^{[m]}$ given in Proposition 4.6, and the formulas (5.3), (4.10), and (4.3), we have that

$$
\begin{aligned}
\Gamma_{m, s, \lambda}(x) & =-\lambda\left(\widehat{\varphi}_{s}^{[m]}(\sinh (x D))\right) \\
& =\frac{1}{2} \sum_{i=0}^{m} \sum_{j \in \mathbb{Z}} \frac{\eta_{i}(x, s-j)}{\sinh (x / 2)}{ }^{a} h_{j}^{(i)}-\frac{1}{2} \frac{\cosh (x / 2)}{\sinh (x / 2)} c_{0} .
\end{aligned}
$$

Now the proposition follows from the definition of exponents and their multiplicities.

Proposition 5.5. Consider $\mathfrak{g}_{+}^{[m]}=c_{\infty}^{[m]}, \mathfrak{g}_{-}^{[m]}=d_{\infty}^{[m]}$ and the embedding $\widehat{\varphi}_{\frac{1}{2}}^{[m], \pm}: \widehat{\mathcal{D}}_{x}^{ \pm} \rightarrow \mathfrak{g}_{ \pm}^{[m]}$. Then the $\mathfrak{g}_{ \pm}^{[m]}$-module $L\left(\mathfrak{g}_{ \pm}^{[m]}, \lambda\right)$ regarded as a $\widehat{\mathcal{D}}_{x}^{ \pm}$-module via $\widehat{\varphi}_{\frac{1}{2}}^{[m]},{ }^{ \pm}$is isomorphic to $L\left(\widehat{\mathcal{D}}_{x}^{ \pm} ; e^{+}, e^{-}\right)$ where $e^{+}, e^{-}$consist of exponents $j \in \mathbb{Z}_{+}$and multiplicities

$$
\sum_{\substack{0 \leqslant i \leqslant m, i \text { even }}} \frac{{ }^{g} h_{j}^{(i)} x^{i}}{i !} \text { and } \sum_{\substack{0 \leqslant i \leqslant m, i \text { odd }}} \frac{{ }^{g} h_{j}^{(i)} x^{i}}{i !}
$$

respectively, where ${ }^{g} h_{j}^{(i)}=0$ for $i$ odd and $g$ represents $c$ or d depending on $\mathfrak{g}_{ \pm}^{[m]}$. 
Proof. We will only need to compute $\Gamma_{m, s, \lambda}(x)$. The rest of the statement is clear, cf. the proof of Proposition 5.4. Recall Remark 4.5(a) and consider the explicit computation of the homomorphism $\widehat{\varphi}_{\frac{1}{2}}^{[m], \pm}: \widehat{\mathcal{D}}_{x}^{ \pm} \rightarrow \mathfrak{g}_{ \pm}^{[m]}$ given in Proposition 4.6. Using (5.3), (4.9), (4.10), and (4.4), we have that

$$
\begin{aligned}
\Gamma_{m, s, \lambda}(x)= & -\lambda\left(\widehat{\varphi}_{\frac{1}{2}}^{[m], \pm}(\sinh (x D))\right) \\
= & \frac{1}{2} \sum_{i=0}^{m} \sum_{j>0} \frac{\eta_{i}(x, j+1 / 2)}{\sinh (x / 2)}{ }^{g} h_{j}^{(i)} \\
& +\frac{1}{2} \sum_{\substack{0 \leqslant i \leqslant m \\
\text { even }}} \frac{\eta_{i}(x, 1 / 2)}{\sinh (x / 2)} g h_{0}^{(i)}-\frac{1}{2} \frac{\cosh (x / 2)}{\sinh (x / 2)} c_{0},
\end{aligned}
$$

which proves the proposition.

Proposition 5.6. Consider the embedding $\widehat{\varphi}_{0}^{[m], \pm}: \widehat{\mathcal{D}}_{x}^{ \pm} \rightarrow \mathcal{L}_{ \pm}^{[m]}$. Then the $\mathcal{L}_{ \pm}^{[m]}$-module $L\left(\mathcal{L}_{ \pm}^{[m]}, \lambda\right)$ regarded as a $\widehat{\mathcal{D}}_{x}^{ \pm}$-module via $\widehat{\varphi}_{0}^{[m], \pm}$ is isomorphic to $L\left(\widehat{\mathcal{D}}_{x}^{ \pm} ; e^{+}, e^{-}\right)$where $e^{+}, e^{-}$consist of exponents $-j-\frac{1}{2}$ with $j \in \mathbb{Z}_{+}$and multiplicities

$$
\sum_{\substack{0 \leqslant i \leqslant m, i \text { even }}} \frac{{ }^{ \pm} h_{j}^{(i)} x^{i}}{i !} \text { and } \sum_{\substack{0 \leqslant i \leqslant m, i \text { odd }}} \frac{{ }^{ \pm} h_{j}^{(i)} x^{i}}{i !},
$$

respectively.

Proof. Recall Remark 4.5(a) and consider the explicit computation of the homomorphism $\widehat{\varphi}_{0}^{[m], \pm}: \widehat{\mathcal{D}}_{x}^{ \pm} \rightarrow \mathcal{L}_{ \pm}^{[m]}$ obtained in Proposition 4.6. Using (5.3), (4.9), (4.10), and (4.5), we have that

$$
\begin{aligned}
\Gamma_{m, s, \lambda}(x) & =-\lambda\left(\widehat{\varphi}_{0}^{[m], \pm}(\sinh (x D))\right) \\
& =\frac{1}{2} \sum_{i=0}^{m} \sum_{j \geqslant 0} \frac{\eta_{i}(x,-j)}{\sinh (x / 2)}{ }^{ \pm} h_{j}^{(i)}-\frac{1}{2} \frac{\cosh (x / 2)}{\sinh (x / 2)} c_{0},
\end{aligned}
$$

which proves the proposition.

Take an irreducible quasifinite highest weight $\widehat{\mathcal{D}}_{x}^{ \pm}$-module $L\left(\widehat{\mathcal{D}}_{x}^{ \pm}, \lambda\right)$ with central charge $\bar{c}$ and

$$
\Gamma_{\lambda}(x)=\frac{\phi_{\lambda}(x)}{2 \sinh (x / 2)},
$$

where $\phi_{\lambda}(x)$ an even quasipolynomial with $\phi_{\lambda}(0)=0$. We will write

$$
\phi_{\lambda}(x)+\cosh (x / 2) \bar{c}=\sum_{s \in \mathbb{C}} \sum_{i=1}^{m_{s}} a_{s, i} \eta_{i}(x, s),
$$

where $a_{s, i} \in \mathbb{C}$ and $a_{s, i} \neq 0$ for only finitely many $s \in \mathbb{C}$. Since, by definition of $\eta_{i}$, we have that $\eta_{i}(x,-s)=(-1)^{i} \eta_{i}(x, s+1)$, to avoid ambiguities in the expression of $\phi_{\lambda}(x)$ above, we will choose the parameter $s$ following these rules: when $s \in \mathbb{Z}$ we require $s \leq 0$; when $s \in \mathbb{Z}+\frac{1}{2}$, we ask $s \leqslant \frac{1}{2}$; when $s \notin \mathbb{Z} / 2$, we require that $\operatorname{Im} s>0$ if $\operatorname{Im} s \neq 0$ or $s-[s]<\frac{1}{2}$ if $s \in \mathbb{R}$, where $\operatorname{Im} s$ is the imaginary part of $s$, and $[s]$ denotes the biggest integer smaller than $s$, respectively.

Decompose the set $\left\{s \in \mathbb{C} \mid a_{s, i} \neq 0\right.$ for some $\left.i\right\}$ into a disjoint union of equivalence classes under the equivalence relation $s \sim s^{\prime}$ if and only if $s= \pm s^{\prime}(\bmod \mathbb{Z})$. Pick a representative $s$ in an equivalence class $S$ such that $s=0$ if the equivalence class is in $\mathbb{Z}$ and $s=\frac{1}{2}$ if the equivalence class is in $\mathbb{Z}+\frac{1}{2}$. Let $S=\left\{s, s-k_{1}, s-k_{2}, \ldots\right\}$ be such an equivalence class and take $m=\max _{s \in S} m_{s}$. Put $k_{0}=0$. It is easy to see that if $s=0$ or $\frac{1}{2}$, then $k_{i} \in \mathbb{N}$.

We associate with $S$ the $\mathfrak{g}_{ \pm}^{[m]}$-module $L_{S}^{[m]}\left(\mathfrak{g}_{ \pm}^{[m]}, \lambda_{S}\right)$ in the following way: if $s \notin \mathbb{Z} / 2$, let ${ }^{a} h_{k_{r}}^{(i)}=$ $a_{s+k_{r}, i}$ with $i=0, \ldots, m_{s}$ and $r=0,1,2, \ldots$ We associate with $S$ the $\widehat{\mathfrak{g}}_{\infty}^{[m]}$-modules $L_{S}^{[m]}\left(\widehat{\mathfrak{g l}}_{\infty}^{[m]}, \lambda_{S}\right)$ 
with central charges and labels

$$
c_{i}=\sum_{k_{r}}{ }^{a} h_{k_{r}}^{(i)}, \quad{ }^{a} \lambda_{j}^{(i)}=\sum_{k_{r} \geqslant j}\left({ }^{a} h_{k_{r}}^{(i)}-\delta_{k_{r}, 0} c_{i}\right) .
$$

If $s=\frac{1}{2}$, let ${ }^{g} h_{k_{r}}^{(i)}=a_{\frac{1}{2}+k_{r}, i}$, with $i=0, \ldots, m_{\frac{1}{2}}$ and $r=0,1,2, \ldots$ We associate with $S$ the $\mathfrak{g}_{ \pm}^{[m]}$-module $L_{S}^{[m]}\left(\mathfrak{g}_{ \pm}^{[m]}, \lambda_{S}\right)$ with central charges and labels

$$
c_{i}=\sum_{k_{r}}{ }^{g} h_{k_{r}}^{(i)}(i \text { even }), c_{i}=0 \text { (i odd), }{ }^{g} \lambda_{j}^{(i)}=\sum_{k_{r} \geqslant j}{ }^{g} h_{k_{r}}^{(i)},
$$

where $\mathfrak{g}_{+}^{[m]}=c_{\infty}^{[m]}, \mathfrak{g}_{-}^{[m]}=d_{\infty}^{[m]}$ and $g$ represents $c$ or $d$ depending on $\mathfrak{g}_{ \pm}^{[m]}, j \in \mathbb{N}, i=0, \ldots, m_{\frac{1}{2}}$. Similarly if $s=0,{ }^{ \pm} h_{k_{r}}^{(i)}=a_{k_{r}, i}$, with $i=0, \ldots, m_{0}$ and $r=0,1,2, \ldots$ We associate with $S$ the $\mathcal{L}_{ \pm}^{[m]}$-module $L_{S}^{[m]}\left(\mathcal{L}_{ \pm}^{[m]}, \lambda_{S}\right)$ with central charges and labels

$$
c_{i}=\sum_{k_{r}}{ }^{ \pm} h_{k_{r}}^{(i)}, \quad{ }^{ \pm} \lambda_{j}^{(i)}=\sum_{k_{r} \geqslant j}\left({ }^{ \pm} h_{k_{r}}^{(i)}-\delta_{k_{r}, 0} c_{i}\right) .
$$

Denote by $\left\{s_{1}, s_{2}, \ldots, s_{N}\right\}$ a set of representative of equivalence classes in the set $\left\{s \in \mathbb{C} \mid a_{s, i} \neq\right.$ 0 for some $i\}$. By Theorem 5.3, the $\widehat{\mathcal{D}}_{x}^{ \pm}$-module $L_{\bar{s}}^{[\bar{m}]}\left(\mathfrak{g}^{[m]}, \bar{\lambda}\right)$ is irreducible for $\bar{s}=\left(s_{1}, \ldots, s_{N}\right)$ such that $s_{i} \in \mathbb{Z}$ implies $s_{i}=0, s_{i} \in \mathbb{Z}+\frac{1}{2}$ implies $s_{i}=\frac{1}{2}$ and $s_{i} \neq \pm s_{j}(\bmod \mathbb{Z})$ for $i \neq j$. Then we have

$$
\Gamma_{\bar{m}, \bar{s}, \bar{\lambda}}(x)=\sum_{i} \Gamma_{m_{i}, s_{i}, \lambda_{i}}(x), \quad c=\sum_{i} c_{0}^{(i)} .
$$

Using Theorem 5.3 and Propositions 5.4, 5.5, and 5.6, we have proved the following result.

Theorem 5.7. Let $V$ be an irreducible quasifinite highest weight $\widehat{\mathcal{D}}_{x}^{ \pm}$-module with highest weight $\lambda$, central charge $\bar{c}$, and

$$
\Gamma_{\lambda}(x)=\frac{\phi_{\lambda}(x)}{2 \sinh (x / 2)}
$$

with $\phi_{\lambda}(x)$ an even quasipolynomial such that $\phi_{\lambda}(0)=0$, which is written in the form (5.4). Then $V$ is isomorphic to the tensor product of the modules $L_{S}^{[m]}\left(\mathfrak{g}^{[m]}, \lambda_{S}\right)$ with distinct equivalence classes $S$.

Remark 5.8. A different choice of the representative $s \notin \mathbb{Z} / 2$ has the effect of shifting $\widehat{\mathfrak{g}}_{\infty}^{[\mathrm{m}]}$ via the automorphism $v^{i}$ for some $i$. It is easy to see that any irreducible quasifinite highest weight module $L\left(\widehat{\mathcal{D}}_{x}^{\mathcal{O}, \pm}, \lambda\right)$ can be obtained as above as a unique way up to the shift $v$.

\section{ACKNOWLEDGMENTS}

The authors were supported in part by grants of Conicet, Foncyt-ANPCyT, and Secyt-UNC (Argentina).

${ }^{1}$ Awata, H., Fukuma, M., Matsuo, Y., and Odake, S., "Subalgebras of $W_{1+\infty}$ and their quasi-finite representations," J. Phys. A 28, 105-112 (1995).

${ }^{2}$ Bloch, S., "Zeta values and differential operators on the circle," J. Algebra 182, 476-500 (1996).

${ }^{3}$ Boyallian, C., Kac, V. G., Liberati, J. I., and Yan, C., "Quasifinite highest weight modules over the Lie algebra of matrix differential operators on the circle," J. Math. Phys. 39, 2910-2928 (1998).

${ }^{4}$ Boyallian, C. and Liberati, J. I., "Representations of symplectic type subalgebra of $W_{\infty}$," J. Math. Phys. 44, 2192-2205 (2003).

${ }^{5}$ Frenkel, E., Kac, V. G., Radul, A., and Wang, W., " $\mathcal{W}_{1+\infty}$ and $\mathcal{W}\left(g l_{N}\right)$ with central charge N," Commun. Math. Phys. 170, 337-357 (1995).

${ }^{6}$ Kac, V. G. and Liberati, J. I., "Unitary quasifinite representations of $W_{\infty}$," Lett. Math. Phys. 53, 11-27 (2000).

${ }^{7}$ Kac, V. G. and Peterson, D. H., "Spin and wedge representations of infinite-dimensional Lie algebras and groups," Proc. Natl. Acad. Sci. U.S.A. 78, 3308-3312 (1981).

${ }^{8}$ Kac, V. G. and Radul, A., "Quasifinite highest weight modules over the Lie algebra of differential operators on the circle," Commun. Math. Phys. 157, 429-457 (1993).

${ }^{9}$ Kac, V. G. and Radul, A., "Representation theory of the vertex algebra $W_{1+\infty}$, , Transform. Groups 1, 41-70 (1996).

${ }^{10}$ Kac, V. G., Wang, W., and Yan, C., "Quasifinite representations of classical Lie subalgebras of $W_{1+\infty}$," Adv. Math. 139, 56-140 (1998). 